\title{
Experiments and Computations on Abstractions of Perching
}

\author{
Kenneth Granlund ${ }^{1}$, Michael $\mathrm{OL}^{2}$, Daniel Garmann ${ }^{3}$ and Miguel Visbal ${ }^{4}$ \\ U.S. Air Force Research Laboratory, Wright-Patterson Air Force Base, OH 45433, U.S.A. \\ Luis Bernal ${ }^{5}$ \\ University of Michigan
}

\begin{abstract}
The flight maneuver of perching is abstracted as a linear pitch ramp, with and without a deceleration in the free-stream direction. We consider, first, experimental-computational comparison for flowfield and aerodynamic force coefficients for an SD7003 airfoil pitching from $\alpha=0^{\circ}$ to $45^{\circ}$; and second, an experimental survey of reduced frequency and pivot point for a range of flat plate pitching cases from $0^{\circ}$ to $90^{\circ}$. The computational approach is $3 \mathrm{D}$ Large Eddy Simulation, and the experimental approach is by three degree of freedom electric motion-rig in a water tunnel. Accurate flowfield resolution in deep-stall is seen to require a large spanwise extent of computational domain. Meanwhile, experiment can be plagued with blockage, and dynamic blockage was seen to behave differently than static blockage. Even very low reduced frequencies of motion give lift overshoot beyond static stall, but comparatively large frequencies are necessary before the lift curve slope changes, either due to rate effects or acceleration effects. Moving the pitch pivot point further aft tends to attenuate both lift and drag production, and pitching about the three-quarter chord point cancels the rate-effect, in agreement with quasi-steady linear airfoil theory. Surprisingly, the aerodynamic coefficient history differs little between cases with and without streamwise deceleration, except towards the very end of the motion. The implication is that perching-type of ground tests or computations can be adequately conducted in a steady free-stream.
\end{abstract}

\section{Introduction}

Study of bird landing behavior ${ }^{1}$ and the so-called "perching" behavior has inspired mechanized versions for manmade flight articles ${ }^{2}$. The complexity of natural flyers, including elastic deformations and wing geometry changes at large scale (planform, dihedral) and small scale (manipulation of fine surface features and deployment of stall-control devices ${ }^{1}$ ) render the passage to manmade flight articles difficult. Nevertheless, at a first approximation the maneuver of perching is related to the classical pitch ramp motion, where the angle of attack varies fairly quickly over a large amplitude. Such pitch ramps produce not only the usual departure from attached flow, in the sense of leading edge vortex growth and shedding, but also very large lift coefficients and lift histories with explicit dependency on motion rate. Graham and Strickland ${ }^{3}$, for example, proposed an effective leading edge angle of attack to unify results at various pitch pivot points, and scaled the lift coefficient by the reduced frequency of pitch, to elucidate rate-dependency.

The key difference between perching and ramp motions in classical unsteady aerodynamics is that in the latter the free-stream velocity is constant during the maneuver, while in perching there is a flight trajectory beginning from a cruise-like condition and terminating in landing. To properly model this in a ground test facility, one needs to reduce the effective free stream by the end of the maneuver to zero.

In this work, we seek to extend the wind tunnel measurements of Reich et al. ${ }^{2}$, for a maximally rudimentary configuration of a wall-to-wall flat plate and a wall-to-wall SD7003 airfoil, across a range of angles of attack, reduced frequencies, pivot point locations, and Reynolds numbers. We compare constant free-stream with a freestream decelerating to zero, and correlate direct force measurements with flow visualization by dye injection. The computation focuses on the reduced frequency $\mathrm{K}=c \dot{\theta} / 2 U_{\infty}=0.03$ for the SD7003 airfoil at $\mathrm{Re}=50 \mathrm{~K}$, with angle of attack range of $0^{\circ}$ to $45^{\circ}$. The experiment covers this case and also considers parameter studies with a flat plate, primarily at $\operatorname{Re}=20 \mathrm{~K}$, angle of attack range from $0^{\circ}$ to $90^{\circ}$, and reduced frequency range from 0.0025 through 0.20 .

\footnotetext{
${ }^{1}$ Post-Doctoral Scholar, Air Vehicles Directorate

${ }^{2}$ Senior Aerospace Engineer, Air Vehicles Directorate, Michael.Ol@wpafb.af.mil

${ }^{3}$ Aerospace Engineering Graduate Student

${ }^{4}$ Senior Aerospace Engineer, Air Vehicles Directorate

${ }^{5}$ Associate Professor, Department of Aerospace Engineering.
} 


\section{Experimental Setup}

\section{Facility and Motion Mechanism}

The U.S. Air Force Research Laboratory's Horizontal Free-surface Water Tunnel has 4:1 contraction and $46 \mathrm{~cm}$ wide by $61 \mathrm{~cm}$ high test section, and is fitted with a three degree of freedom electric rig enabling independent control of pitch or rotation, plunge or heave, and "surge" or streamwise-aligned translation. Tunnel free-stream speed range is $3-45 \mathrm{~cm} / \mathrm{s}$. A photograph of the tunnel, schematic of two motors of the pitch-plunge rig and installation of the free-to-pivot flat plate in the tunnel are shown in Figure 1. In the photograph, the model is inside the test section, but the test section glass sidewalls are not visible. More detail on the rig operation and discussion of experimental error is given in $\mathrm{Ol}$ et al. ${ }^{4}$

Water tunnel turbulence intensity ( $\mathrm{u}$ and $\mathrm{v}$ components) as determined from PIV free-stream data and confirmed by laser-Doppler velocimetry, is estimated at $0.4 \%$ at $U_{\infty}=30-40 \mathrm{~cm} / \mathrm{s}$. A surface skimmer plate at the entrance to the test section and a sealed lid of the intake plenum (visible as plywood cover in Figure 1) damp sloshing in the tunnel, that could otherwise have been be excited as a first-mode shallow water wave, and would have resulted in low-frequency $(\sim 0.16 \mathrm{~Hz})$ sinusoidal variation in $U_{\infty}$. More detail on the facility is given in $\mathrm{Ol}$ et al. ${ }^{5}$

The 3-component oscillator rig is controlled through a Galil DMC 4040 Ethernet controller. Pitch and plunge are made possible by a pair of motors mounted vertically on a plate above the tunnel test section, shown in the middle portion of Figure 1. Each motor actuates a vertical "plunge rod", which connects via a bushing to a coupler piece. The coupler piece is imbedded in the model ${ }^{6}$. The flat plate and airfoil models are wall-to-wall with nominal $0.5 \mathrm{~mm}$ gap on each tip, and $15.24 \mathrm{~cm}$ chord. The plate is $2 \%$ thick and has round edges.
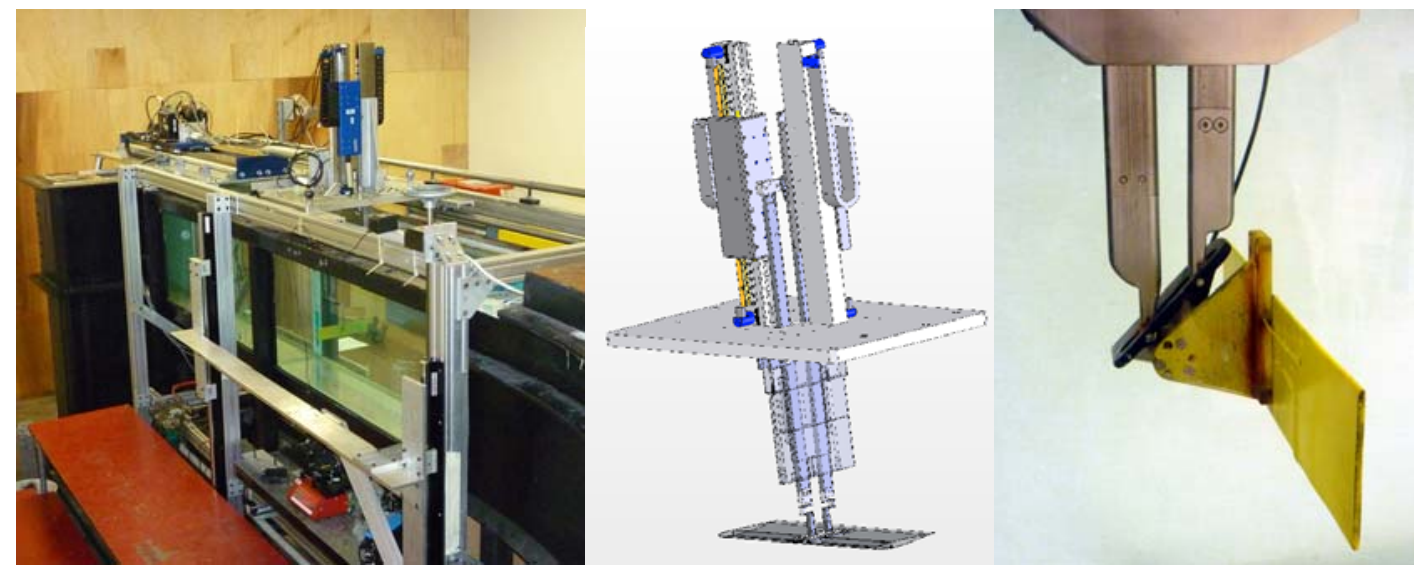

Figure 1. Test section and motion apparatus mounted above test section of the AFRL Horizontal Freesurface Water Tunnel (left); schematic of pitch/plunge rig's vertical-axis motors (middle); and 3"-chord flat-plate wall-to-wall model. The force balance is integrated into the plunge rod coupler (right).

The upstream plunge rod of the pitch-plunge portion of the mechanism is constrained to move purely vertically, whereas the downstream plunge rod is allowed to pivot in the test section vertical plane of symmetry. The desired angle of incidence and vertical position time history of the model are converted to position commands for each linear motor. The pitch pivot point can be varied by suitable choice of phase and amplitude difference in trajectory of front or rear plunge rod. For all cases where the pitch pivot point is not coincident with the bushed end of the front plunge rod, there will be a parasitic streamwise displacement of the model, which would be unavoidable unless the front plunge rod were to be allowed to pivot similarly to the downstream one. This is removed using the third degree of freedom, surge, which also actuates the fore and aft translating motion of the model. Surge is achieved using a larger linear motor mounted horizontally aft of the pitch-plunge carriage, with 48" peak-to-peak stroke and nominal speed up to $1 \mathrm{~m} / \mathrm{s}$. Perching trajectory can be effected by beginning a test sequence with the model at the aft end of the test section, accelerating rapidly to a steady forward speed, against the free-stream. This is the "cruise" condition prior to initiating the perching maneuver. Then the model begins to decelerate, reaching 
some position towards the extreme upstream end of the test section, stopping, and reversing direction, until towards the aft end of the test section the towing speed equals the free-stream speed, whereupon there is no longer a relative motion in the streamwise direction. This is continued briefly, until the model comes to rest in the lab-frame before running out of test section length. Such a procedure effectively doubles the amount of convective-times that the perching maneuver can occupy, vs. a simple one-direction tow commencing at the test section upstream end and ending at the aft end. This is useful for the lower reduced-frequencies of perching, where the maneuver occurs over many chord-lengths of motion in the streamwise relative direction. It also becomes possible to operate at larger Reynolds number than what would have been possible with the tunnel running alone, at its maximum speed.

\section{Velocimetry Methods}

Particle image velocimetry measurements were taken with a PCO 400011 Mpix camera run in single A-D mode, triggered from an external pulse train derived from the position encoder of the motion rig's surge motor, thus allowing for selection of motion phase at which to acquire data. Velocity data and the derived vorticity data are not ensemble-averaged, but are all from one motion sequence, separated by approximately $5.6^{\circ}$ of displacement in pitch. Vorticity was calculated by explicit differentiation of cubic spline fits to the velocity field (as discussed by Willert and Gharib ${ }^{7}$ ). PIV resolution was typically 160 pixels $/ \mathrm{cm}$., which for 32x32-pixel windows with $16 \times 16$ overlap gives approximately 50 vectors per chord length. Because of laser reflections from the model surface (carbon fiber, spray-painted flat-black for PIV) and lack of corrections for PIV windows which at least partially intersect with the model surface, data in the first row of PIV windows adjacent to the airfoil surface are not reliable. Light sheet thickness was approximately $1.5 \mathrm{~mm}$ max, though the large field of coverage (up to $45 \mathrm{~cm}$ across in some cases) makes precise collimation of the light sheet difficult. To obtain the "most 2-dimensional" flowfield, the PIV light sheet was placed at the 3/4-span location; that is, approximately halfway between the plunge rods and the tunnel wall.

Dye injection is by a positive-displacement pump with a prescribed volumetric infusion rate, connecting to a set of $0.5 \mathrm{~mm}$ internal-diameter rigid lines glued to the surface of the plate ${ }^{4}$. For visualizing leading edge vortices, the dye lines run along the plate leading edge, firing outboard. For visualizing the wake, the dye lines exit in the streamwise direction, terminating just upstream of the trailing edge. Typically dye exit speed is $\sim 0.30$ of the freestream speed, balancing desire for high dye concentration with minimal flowfield disruption. The dye fluid consists of approximately $70 \%$ blue food coloring, $20 \%$ alcohol and $10 \%$ coffee cream, for approximate density matching with that of the ambient water. Dye ports were also placed at the $3 / 4$-span location.

\section{Force Measurement}

Force measurements were with an ATI Nano-25 waterproof 6-component balance (right-hand image in Figure 1), oriented to align its maximally sensitive channels with the longitudinal forces and moments (lift, drag and pitching moment). For maximal force balance sensitivity and reduction of vibrations, the balance should be near the model's center of pressure. This however introduces unacceptable interference. The aerodynamically cleaner arrangement is an aft sting, where the model is mounted some distance ahead of the balance, and the balance is integrated into a housing that connects to the two vertical plunge rods. Here one must be careful about the stiffness of the sting and the balance itself. The "apparent mass" of the water accelerated along with the model - essentially a circular cylindrical slug of water with length the same as that of the model, and diameter equal to the model's chord - can be 10 times larger than that of the model, sting, and metric portion of the balance. Therefore, in direct contradistinction with wind tunnel unsteady aerodynamics testing practice, it is not necessary to build a light model or sting, and instead the preference is for high strength at the expense of mass.

Position accuracy for the SD7003 airfoil was $\pm 0.2^{\circ}$, based on balance stiffness and rig dynamic following error. For the carbon fiber plates (6" and 3" chord), position accuracy was far worse - about $\pm 1^{\circ}$ - because as the carbon absorbs water from weeks of immersion, it deflects, introducing a spanwise twist and sagging.

\section{Computational Approach}

\section{Method Definition}

The compressible Navier-Stokes equations are solved using the implicit, large-eddy solver FDL3DI ${ }^{8,9}$ High-order spatial discretization of the governing equations is achieved using a compact finite difference formulation as described by Lele ${ }^{10}$. For a scalar quantity, $\phi$, such as a metric, flux component of flow variable, the

spatial derivative, $\phi^{\prime}$, is determined along a coordinate line in the transformed plane through the tridiagonal system, 


$$
\alpha \phi_{i-1}^{\prime}+\phi_{i}^{\prime}+\alpha \phi_{i-1}^{\prime}=a \frac{\phi_{l+1}-\phi_{l-1}}{2}+b \frac{\phi_{i+z}-\phi_{l-z}}{2}
$$

where the coefficients, $\alpha, a$, and $b$, are chosen based on matching coefficients from the Taylor series expansions of each quantity about point $i$, to achieve the desired order of accuracy. Up to sixth-order accuracy can be achieved with proper choice of coefficients, namely, $\alpha=1 / 3, a=14 / 9$, and $b=1 / 9$, which were used here. At boundary points, higher-order one-sided differences retaining the tridiagonal form are used ${ }^{8,9}$.

Unlike the standard LES approach, no additional sub-grid stress models are used to model the small scale structures associated with transitional or turbulent flows. Instead, a high-order, low-pass spatial filtering operator ${ }^{12}$ is applied to the conserved variables to discriminately eliminate only the poorly resolved, high-frequency content of the solution. If a typical component of the solution vector is denoted by $\phi$, then filtered values, $\bar{\phi}$, at interior points of the domain in the transformed plane are given by

$$
\alpha_{f} \hat{\phi}_{i-1}+\tilde{\phi}_{i}+\alpha_{f} \hat{\phi}_{i-1}=\sum_{n=0}^{N} \frac{a_{n}}{2}\left(\phi_{i+n}+\phi_{i-n}\right)
$$

where the explicit stencil size, $N$, on the right-hand side is determined by the desired order of accuracy. This formulation is based on templates proposed by Lele ${ }^{10}$ and Albert ${ }^{11}$, and with proper choice of coefficients, $a_{n}$, provides a $\mathbf{2} N^{\text {th }}$-order formula on a point stencil. As with the spatial discretization, these coefficients are found by eliminating the Taylor coefficients up to the desired order of accuracy ${ }^{12}$. The coefficient, $\alpha_{f}$, is left as a free variable for additional control of the higher frequency dissipation. A value close to 0.5 provides very little dissipation of the highest frequencies. For the cases presented here, $\alpha_{f}=\mathbf{0 . 3}$

Time-marching is achieved through the iterative, implicit approximately-factored time integration method of Beam and Warming ${ }^{13}$. This method has been simplified through the diagonalization of Pulliam and Chausse ${ }^{14}$ and supplemented with the use of Newton-like sub-iterations to achieve second-order accuracy.

\section{Implementation of the Computational Method}

The original sharp trailing edge of the SD7003 airfoil was rounded with a circular arc radius, $r / c \approx 0.0004$, allowing the use of an O-grid topology as depicted in Figure 2.

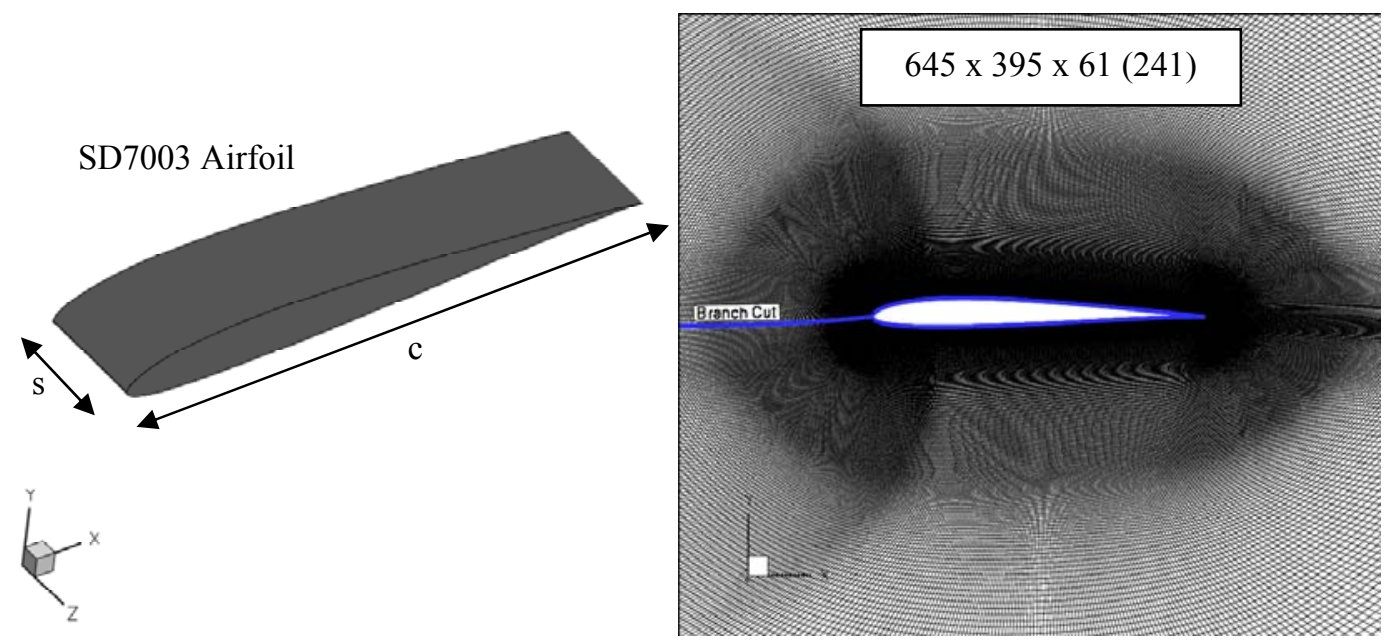

Figure 2: SD7003 Grid Topology

On the airfoil surface, 645 grid points were distributed, to allow for clustering near the leading and trailing edges. The initial spacing normal to the airfoil surface was $0.00005 c$ with 395 grid points stretching outward ending in a circular boundary nearly 100 chords two span-to-chord ratios were considered: 0.2 and 0.8 . In each, the spacing in the spanwise direction was kept constant at $0.0033 \mathrm{c}$, chosen based on the average spacing around the airfoil surface. This resulted in 61 and 241 grid points in the spanwise direction for the 0.2 and 0.8 cases, respectively.

A no-slip, adiabatic boundary condition was prescribed on the airfoil surface, along with a third-order extrapolated zero normal pressure gradient. A uniform stream was enforced at the farfield boundary, where it should be noted that the grid points are very rapidly stretched. The stretching along with the low-pass filter provides 
a means of preventing spurious reflections 15 . The three-dimensional cases use spanwise boundary conditions. To simulate airfoil pitch, the entire domain is rotated rigidly without deformation.

\section{Results}

We divide the following into seven subjects:

1. Comparison of experimental and computational results for the flowfield (principally, vorticity) of the SD7003 airfoil at $\mathrm{Re}=50 \mathrm{~K}$, in linear pitch from $\alpha=0^{\circ}$ to $\alpha=45^{\circ}$, with the aforementioned acceleration transients at startup and stopping.

2. Comparison of experimental and computational results for the SD7003 lift and drag coefficient history for $\alpha$ $=0^{\circ}$ to $\alpha=45^{\circ}$.

3. Flat plate vs. SD7003 airfoil, and comparison of 3"-chord and 6"-chord flat plates, to ascertain the role of static and dynamic blockage in the water tunnel, as concerns lift and drag coefficient history.

4. Parameter study of reduced frequency in pitch, for the 3"-chord flat plate.

5. Parameter study of the various pivot point locations, for the 3 "-chord flat plate.

6. Comparison of 3"-chord flat plate cases with and without deceleration in the free-stream direction.

7. Study of connection between flowfield phenomena and trends in aerodynamic force history for the various flat-plate cases, including discussion of blockage effects on the flowfield vs. the aerodynamic force history.

\section{Computational-Experimental Comparison of SD7003 Flowfields}

Experiment includes dye injection and PIV, while the computation is of three types: 2D, $0.2 \mathrm{c}$-spanwise extent, and 0.8c-spanwise extent. In Figure 3, experiment and computation both show out-of plane vorticity component, normalized by airfoil chord and free-stream speed. This is compared with dye concentration images in the experiment. Dye concentration - a scalar quantity - seems to comport well with color-intensity of the normalized vorticity out of plane component, but of course is agnostic to sign (clockwise or counterclockwise rotation). At the phase of motion where an LEV is clearly present - the $5^{\text {th }}$ row of images in Figure 3 - heavy dye concentrations marks the LEV outline. In subsequent phases of motion, dye marks the shear layers emanating from the leading edge and trailing edge. The PIV is a single sequence, with no phase averaging, filtering or smoothing and thus large admixtures of fine-structures of positive and negative vorticity.

As expected, inclusion of larger spanwise extent brings the computed vorticity contours closer to the experimental result. Early in the motion, all computational results are very much alike, and experiment does not differ much either. Evidently the Karman-type vortex shedding from the trailing edge and the upstream progression of stagnant/reversed flow from the trailing edge, visible especially in the dye injection, is a $2 \mathrm{D}$ process, and whatever role of turbulence is present, is not dependent on spanwise resolution. As the motion progresses, the deleterious effect of $2 \mathrm{D}$ computation becomes more manifest. The $2 \mathrm{D}$ computation does not resolve the LEV correctly, instead rendering it as a collection of strong but small discrete vortices. The experiment does not show a strong coherent TEV, though there is evidence of a TEV in the $7^{\text {th }}$ and $9^{\text {th }}$ rows of Figure 3 . TEV strength decreases in the computations in going from $2 \mathrm{D}$ to $0.2 \mathrm{c}$ to $0.8 \mathrm{c}$ computation. After the LEV is shed, the experiment evinces what are evidently Kelvin-Helmholz instabilities in the leading-edge shear layer. These are not quite as evident in the computations, though the $0.8 \mathrm{c}$ result shows plausible rendition of such structures in the $8^{\text {th }}$ row of Figure 3 .

Evidently, large separated structures, such as the LEV and vortical activity in the near-wake, depend on a length scale comparable to $0.2 \mathrm{c}$ or even larger. The onset of separation is likely predicted well with a computation of small spanwise extent, or even in 2D - but resolution of the subsequent flowfield history requires large spanwise extent of the computational domain.

\section{Computational-Experimental Comparison of SD7003 Forces}

Having considered the importance of computational spanwise extent to flowfield resolution, we turn to comparison of lift and drag between the experiment and the computations. Figure 4 compares experimental runs at $\mathrm{Re}=20 \mathrm{~K}$ and $50 \mathrm{~K}$ with the three variants of computation. Broadly, the trend in lift is a linear segment up to around $\alpha \sim 35^{\circ}$, followed by nonlinearity and various oscillations. All computations show a kink in the lift curve at around $\alpha=12^{\circ}$. Below this point, all computations essentially agree, with small sinusoidal-type of undulations associable with Karman trailing vortex shedding. Thereafter, 2D computation has oscillations in lift and drag coefficients not present in the other results. These are evidently associable with the formation and shedding of discrete vortices from the airfoil suction side. The $0.2 \mathrm{c}$ and $0.8 \mathrm{c}$ computations show similar results through $\alpha \sim 35^{\circ}$, above which the $0.2 \mathrm{c}$ run predicts a second and higher lift peak, while the $0.8 \mathrm{c}$ run does not. 


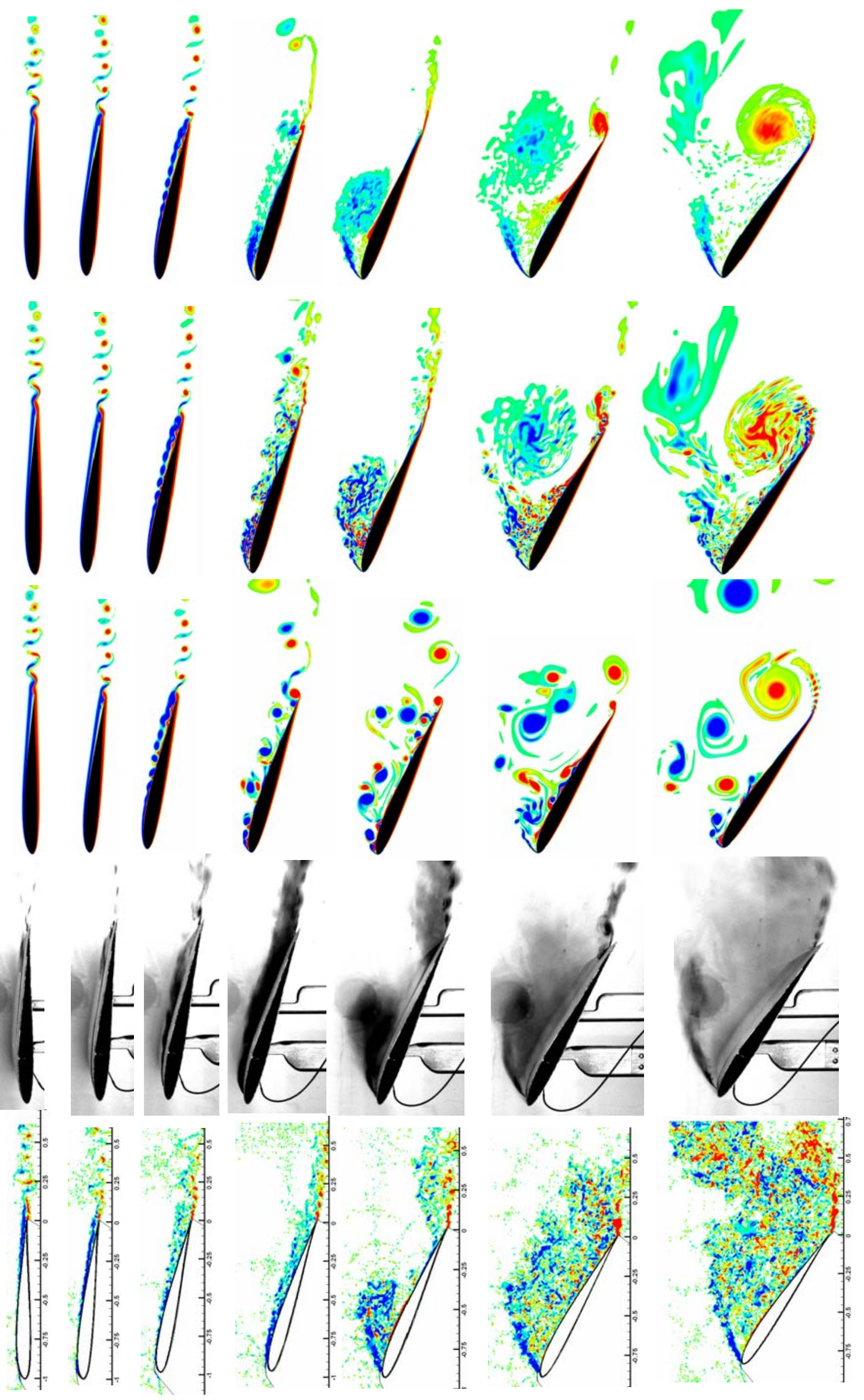



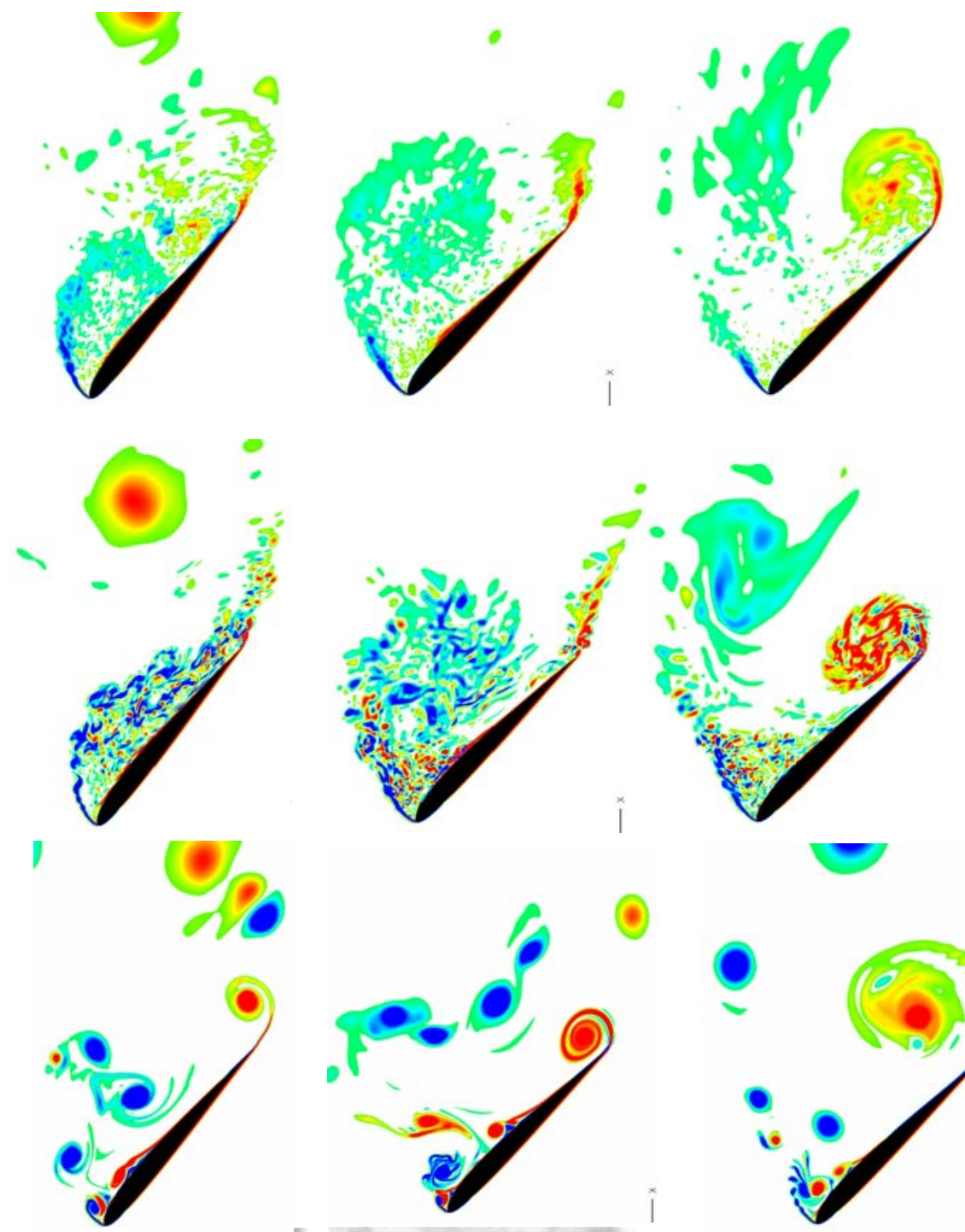

0
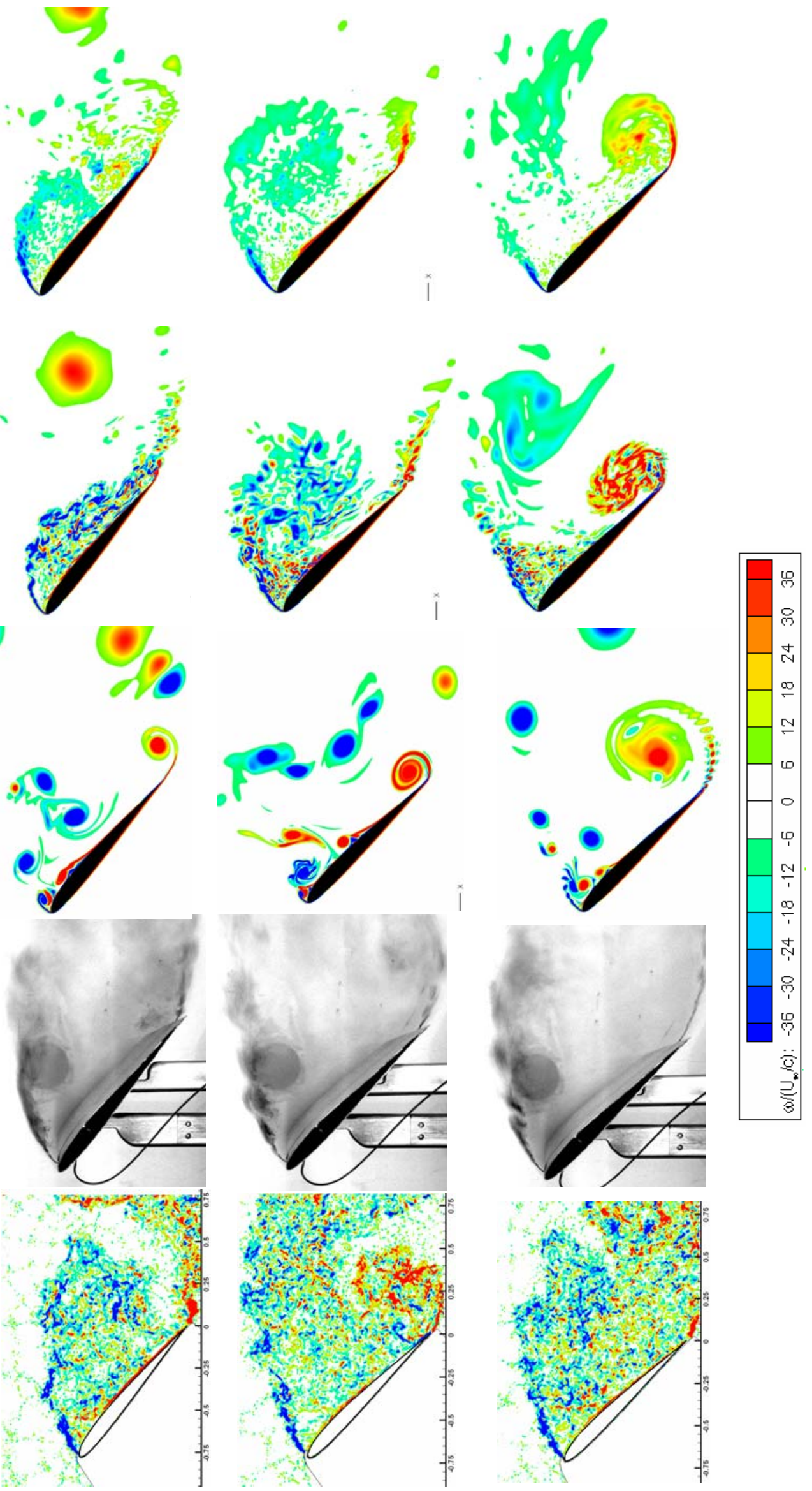

ิํㅇ

产

충

를

豆

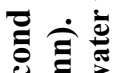

速声

.

.

政

宅 훙

奥要

光

¿ के

送

을

苍 की

됙

를를

产

ฮิ

昰

ఏల

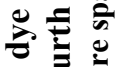

를

部豆

흘 훙 퐁

ํํㅇ

ह

ว

突

守

อั

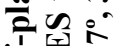

깅

है लि०

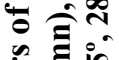

謨

نْ

लि 읍

○ 寻

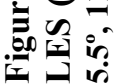


The experiment at $\mathrm{Re}=50 \mathrm{~K}$ generally shows good agreement with the $0.8 \mathrm{c}$ computation, with a smaller lift peak value, but the same stall angle of attack. The experiment has less oscillation of lift than the computations, and a lower peak drag than the computations. At $\mathrm{Re}=20 \mathrm{~K}$, the measured lift curve is offset below the $\mathrm{Re}=50 \mathrm{~K}$ results by approximately $\mathrm{C}_{\mathrm{L}}=0.2$. We somewhat blithely call this a low-Reynolds number effect, as most likely the airfoil experiences a decambering from build-up of laminar separation on the suction side. The zero-alpha lift at $\mathrm{Re}=20 \mathrm{~K}$ is actually slightly negative. Drag however appears to be nearly insensitive to Reynolds number. Evidently, the difference in drag measurements is comparable to balance sensitivity in this range of dynamic pressure, whereas the difference in lift is noticeable because of open laminar separation on the suction side. It is somewhat counterintuitive that the low-alpha lift deficit of the $\mathrm{Re}=20 \mathrm{~K}$ case persists all the way through stall, whence the $\operatorname{Re}=$ $20 \mathrm{~K}$ and $50 \mathrm{~K}$ lift curves are separated by essentially a constant offset. However, it is possible for boundary layer reattachment which occurs in a static sweep of angle of attack, closing a laminar bubble, to be delayed or otherwise inhibited by coupling between the suction-side separation and the dynamics of pitching motion.
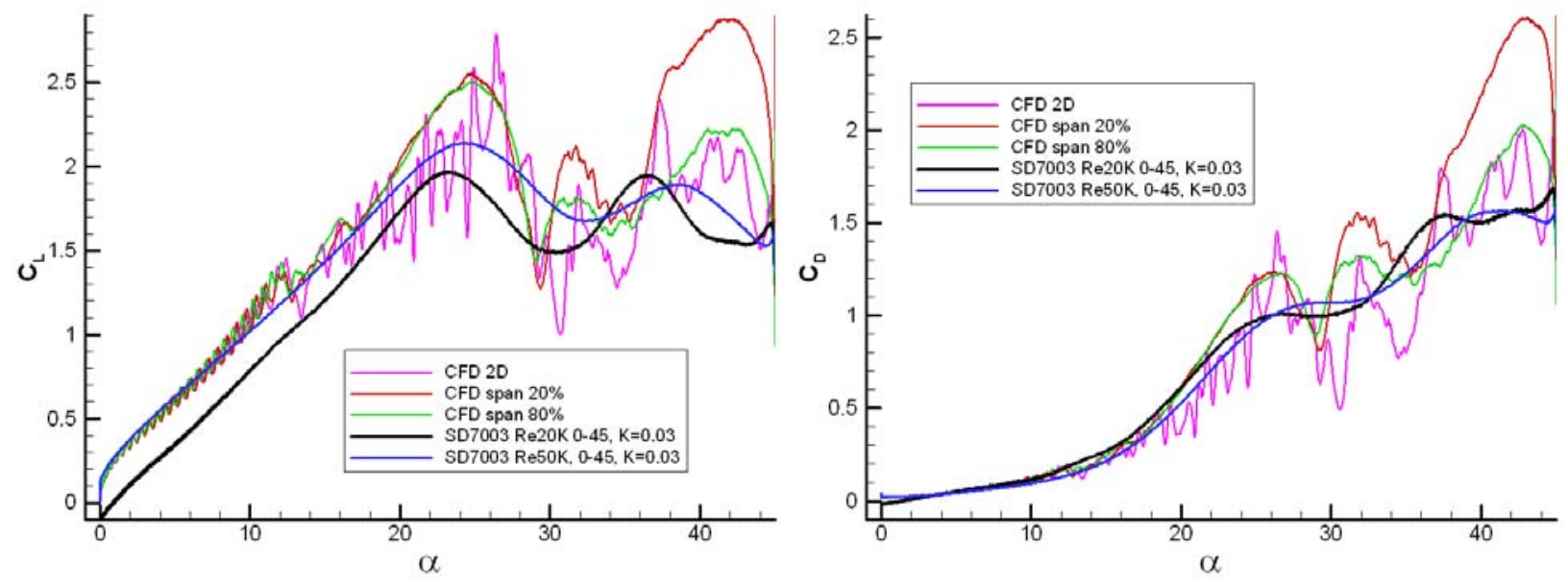

Figure 4. Lift coefficient (left) and drag coefficient (right) for the SD7003 airfoil, $K=0.03$, pitch from $\alpha=0^{\circ}$ to $45^{\circ}$. $R e=$ $50 \mathrm{~K}$ (computation), and $\mathrm{Re}=20 \mathrm{~K}$ and $50 \mathrm{~K}$ (experiment).

For purposes of comparing experiment and a large LES computation that seeks to resolve fine-scale structures, it is useful to consider a transition-dominated case such at the SD7003. But for large parameter studies we wish to deemphasize viscous effects and therefore consider a flat-plate with rounded edges. In the following, we consider the role of blockage - static blockage vs. dynamic blockage. This is important from the viewpoint of assessing deep stall and the reliability of measurements used to construct phenomenological closed-form models for lift and drag as functions of motion parameters.

\section{Airfoil vs. Flat Plate, and Role of Blockage}

An important question in the experiment is the role of blockage, or ratio of the model's length scale (chord) to the facility scale (test section height). Recent work on a linear pitch-ramp-hold-return motion ${ }^{16}$ implied good agreement between experiment and computation for lift coefficient, provided that the experimental data set was divided by a factor of 1.5. Such a factor is intellectually unsatisfying and scientifically specious. We now revisit the likely culprit - blockage - by comparing two flat plates, both wall-to-wall, but one with 6" chord and one with 3" chord. We also broaden the angle of attack range of interest to $0^{\circ} \leq \alpha \leq 90^{\circ}$.

We first compare the SD7003 airfoil and flat plate, both with 6" chord, and presumably identical blockage effects, $R e=20 \mathrm{~K}$ and $50 \mathrm{~K}, \alpha=0^{\circ}-45^{\circ}$ and $\alpha=0^{\circ}-90^{\circ}$ (Figure 5). The flat plate is seen to have very little Redependency, as expected. The plate has less lift at the lowest angles of attack than does the airfoil at $\mathrm{Re}=50 \mathrm{~K}$, with stall occurring slightly earlier, and a weak nonlinearity in lift curve slope over $0^{\circ} \leq \alpha \sim 18^{\circ}$. The plate also has appreciably more drag than the airfoil in the $8^{\circ} \leq \alpha \leq 20^{\circ}$ range, evidently due to more pronounced flow separation on the suction-side. 

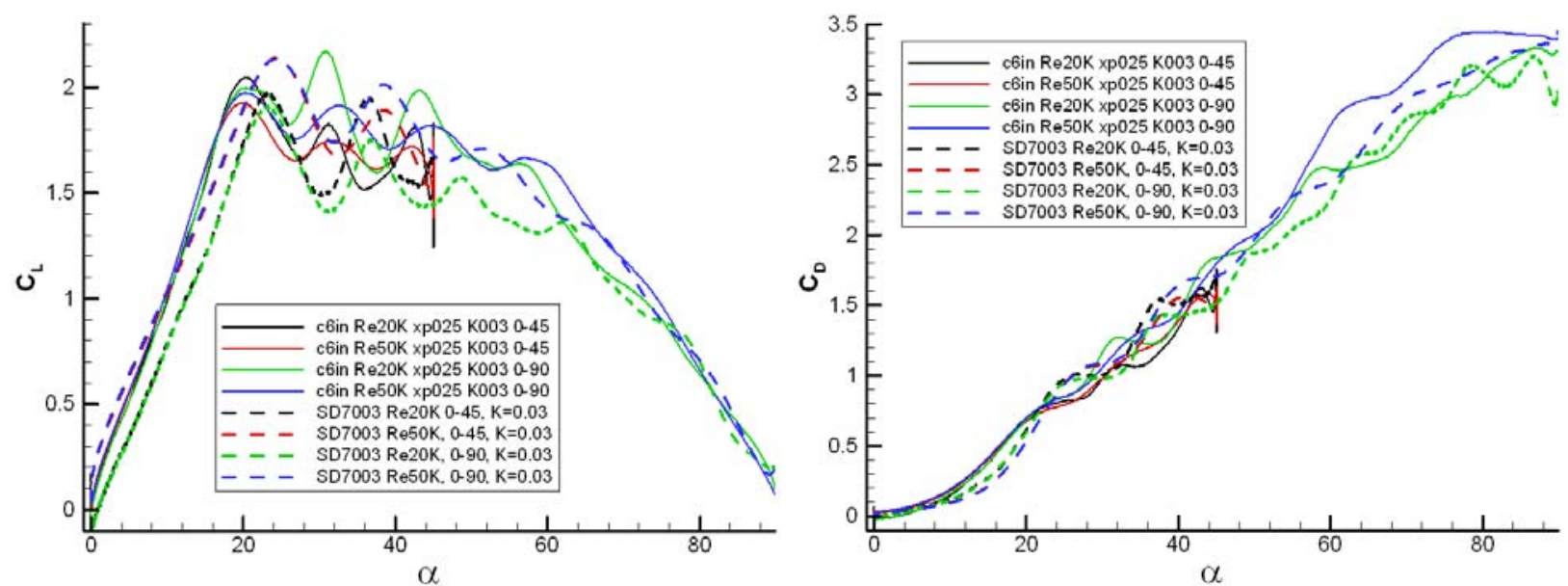

Figure 5. Lift (left) and drag (right) for $\mathrm{SD7003}$ airfoil and flat plate with round edges: $\mathrm{Re}=20 \mathrm{~K}$ and $50 \mathrm{~K}$. $\mathrm{K}=0.03$.

An overlay of $\alpha=0^{\circ}-45^{\circ}$ force history with $\alpha=0^{\circ}-90^{\circ}$ is essentially a repeatability test for $\alpha \leq 40.5^{\circ}$, because for $40.5^{\circ} \leq \alpha \leq 45^{\circ}$, the $\alpha=0^{\circ}-45^{\circ}$ motion has a deceleration towards the motion stopping point . Differences between $0^{\circ}-45^{\circ}$ and $0^{\circ}-90^{\circ}$ runs, for $\alpha \leq 40.5^{\circ}$, when plotted with $\alpha$ as the abscissa, are in variations of shedding after the LEV has been ejected. After the motion has ceased, the effects of vortex shedding will appear for the $\alpha=0^{\circ}-45^{\circ}$ motion as a vertical line segment at $\alpha=45^{\circ}$.

Drag in Figure 5 marches almost monotonically to $\alpha=90^{\circ}$, to the unrealistically high value of $C_{D} \sim 3.5$, with undulations due to bluff-body-type shedding. The typical value of $C_{D}$ in the literature for a flat plate inclined normally to a steady free stream is around $2.0^{17}$. This is further evidence of blockage in the water tunnel.

Figure 6 compares $\mathrm{K}=0.030^{\circ}-90^{\circ}$ pitch sweeps for the 6"-chord and 3"-chord plates. Static data are also given for the two plates. Static data are obtained by slowly stepping through the angle of attack range, holding at the desired value, and time-averaging the measurements over 10 seconds.

Difference between the static 6" plate and 3" plate results is substantial - almost a factor of 2 in lift, and nearly as much in drag. Up to $\alpha \sim 8^{\circ}$, the two results overlap. But at $\alpha \sim 8^{\circ}$ the 3" plate static-stalls, whereas the 6" plate never has an unambiguous stall. For the 3 " plate, the $\alpha=90^{\circ}$ drag coefficient is just over 2.0, in reasonable agreement with the literature.

Dynamic blockage appears to be more benign than static blockage, in the sense that the 6" plate's and 3" plate's $\mathrm{K}=0.003$ curves (cyan and purple curves in Figure 6, respectively) differ less from one another, than do the respective static results. Significant divergence does not arise until sometime after peak lift, occurring at $\alpha>20^{\circ}$ in lift, and at even higher angle of attack in drag. Evidently, the pitching motion suppresses or delays the factors contributing to blockage.
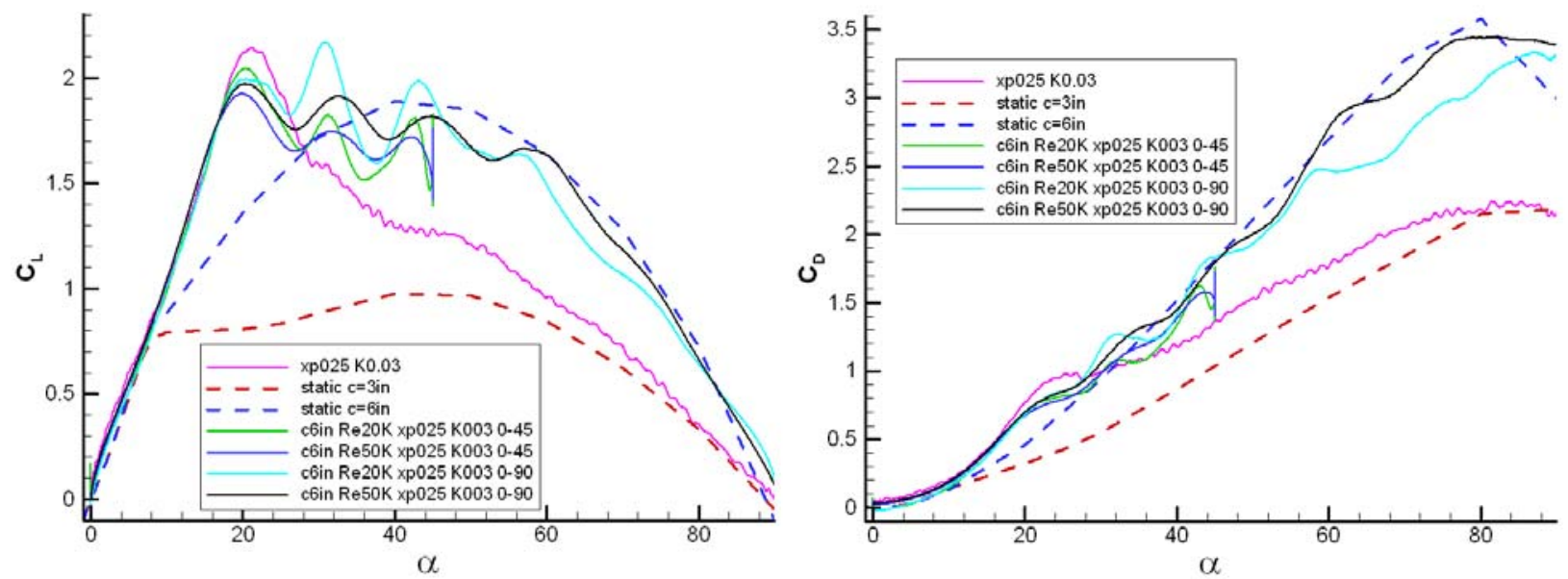

Figure 6. Lift (left) and drag (right) trends for 3" and 6" chord plates, $K=0.03$ and static alpha sweep. 
We rely on the 3" plate's $\alpha=90^{\circ}$ static drag result to regard this model as acceptably blockage-free. In the following we therefore limit the measurements to the 3"-chord plate, and consider a parameter study of reduce frequency (pitch rate) and pitch pivot point.

\section{Role of Pitch Rate}

Here we pursue something of a systematic experimental study of the longitudinal aerodynamic coefficients of a flat plate vs. dimensionless pitch rate, focusing on the pivot point $\mathrm{x} / \mathrm{c}=0.25$, as in all of the foregoing sections. Pitch rates of $\mathrm{K}=0.0025,0.005,0.01,0.02,0.03,0.05,0.075,0.100,0.125,0.150$ and 0.200 are considered in Figure 7, all at $\mathrm{Re}=20 \mathrm{~K}$, and are compared with the aforementioned static sweep. $\mathrm{K}=0.0025$ closely follows the static result, but even $\mathrm{K}=0.005$ departs from static in the sense of stall delay. It is perhaps surprising that even such a slow pitch rate evinces unsteady effects. The lift curve slope follows the static value, and this remains the case for pitch rates up to $\mathrm{K}=0.02$; increasing $\mathrm{K}$ simply delays the stall angle. It is premature to definitively call this a classical dynamic stall, since classical dynamic stall generally refers to the results of a sinusoidal periodic variation of angel of attack. But that said, there is no slope change in the lift curve, and therefore one can with some license call the $\mathrm{K}=0.005$ through 0.02 range the "dynamic stall range" of reduced frequency, whereas $\mathrm{K}<\mathrm{can}$ be dubbed the "quasisteady" region.
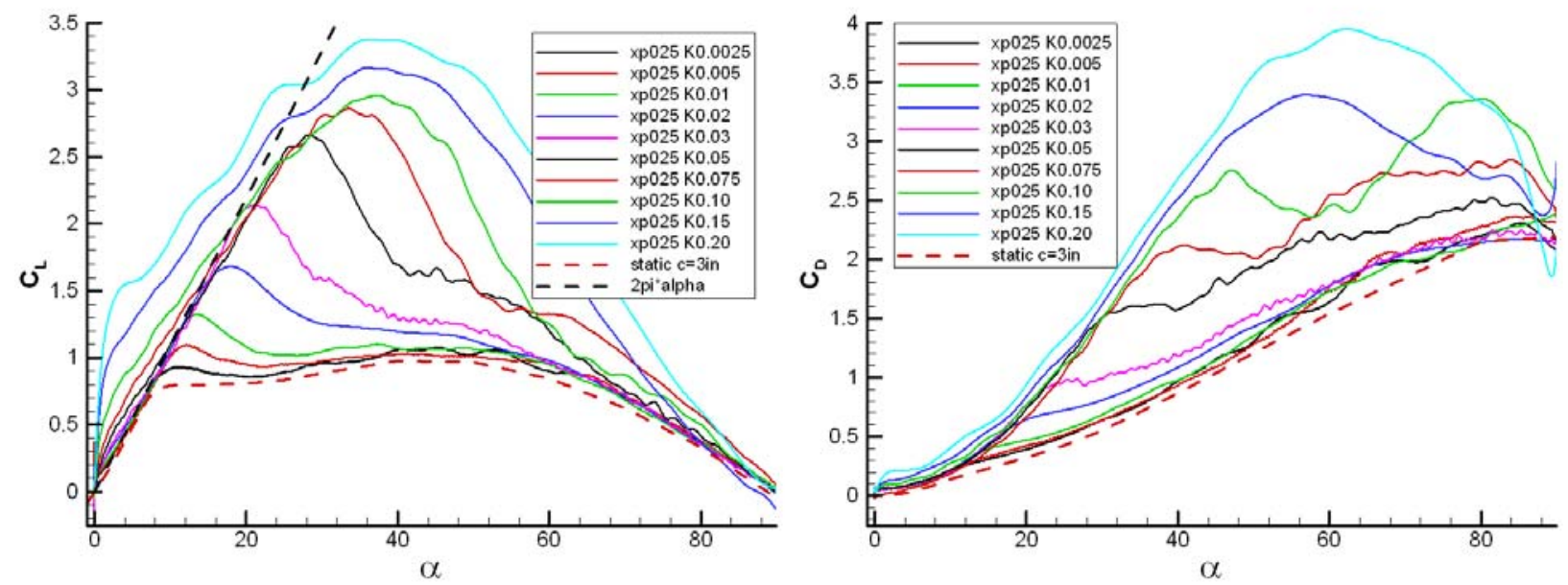

Figure 7. Lift (left) and drag (right) for $c=3$ " flat plate, pivoting at $\mathrm{x} / \mathrm{c}=0.25, \alpha=0^{\circ}-90^{\circ}$, reduced frequency as marked.

For $\mathrm{K}=0.03$ and above, there is a demonstrable lift curve slope change at low angle of attack, and the stall behavior - when it eventually occurs - is broader and smoother than for the aforementioned dynamic stall range of reduced frequency. Evidently the increase in lift at low angle of attack is not due to organized vortical effects, such as a leading edge vortex, since such a structure has not yet had time to form. Instead we surmise the low-alpha increase in lift to depend on the rate of change of angle of attack, in the sense of classical unsteady airfoil theory. We will refer to $\mathrm{K}=0.03$ through 0.1 , approximately, as the rate-dependent range of reduced frequency.

Finally, at the upper bound of the reduced frequencies considered here, there is a clear spike in lift coefficient associated with the pitch-ramp startup transient. The spike occurs strictly over that range of angle of attack during which pitch acceleration is nonzero, and is therefore noncirculatory. The very high apparent lift curve slope at low angle of attack is a linear combination of "lift" proper (circulatory lift, including rate dependency) and the noncirculatory lift. The noncirculatory contribution is in the direction normal to the plate's motion. At low angle of attack, this motion projects predominantly into the lift direction, whereas as $\alpha$ approaches $90^{\circ}$, the platenormal motion projects into the direction of drag. Thus the acceleration in pitch at low $\alpha$ produces a noncirculatory force that manifests itself as a positive increment in lift, while the deceleration in pitch at high $\alpha$ results in a negative increment in drag. Indeed, a drop in drag is seen in the right-hand side of Figure 7 at high $\alpha$ for the faster rates of motion.

Thus it is not the case that there is one approximate value of pitch rate below which the lift response is quasi-steady, and above which it is unsteady. Rather, there are several intervals of pitch rate, each with its own quintessential kind of unsteadiness. 


\section{Role of Pitch Pivot Point}

We now consider pivot point effects, comparing pivoting at $\mathrm{x} / \mathrm{c}=0$ (the leading edge), $0.25,0.50$ and 0.75 . Figure 8 considers these trends for a representative value of moderate reduced frequency and high reduced frequency -0.05 and 0.20 , respectively. As the pivot point is taken to be further aft, the aerodynamic force coefficient magnitude is attenuated across the entire $\alpha$-range, from $0^{\circ}$ through $90^{\circ}$. The higher the reduced frequency, the more pronounced the role of pivot point location. For $\mathrm{K}=0.05$, taking the pivot point further aft slightly reduces the lift at angles of attack below peak lift, and even more slightly reduces the drag. But for $\mathrm{K}=$ 0.20 , the reduction in both lift and drag is very significant. For all cases, the further aft the pivot point, the higher the angle of attack for peak lift coefficient.

We can identify three causes for trends in force coefficient vs. pivot point location. One, the role of LEV development, is discussed further below. Another is the rate-dependency. Quasi-steady airfoil theory predicts zero pitch-rate contribution to lift coefficient for $\mathrm{x} / \mathrm{c}=0.75$ pivot location ${ }^{18}$, and more pitch-rate dependency as the pivot point is taken further from $\mathrm{x} / \mathrm{c}=0.75$. Indeed, the lift curves for $\mathrm{x} / \mathrm{c}=0.75$ pivot in Figure 9 do not show an ever large lift with increasing reduced frequency. Instead, the curves of lift vs. angle of attack when taken as a group evince of kind of maximal envelope, that generally trends below the $C_{L}=2 \pi \alpha$ line.
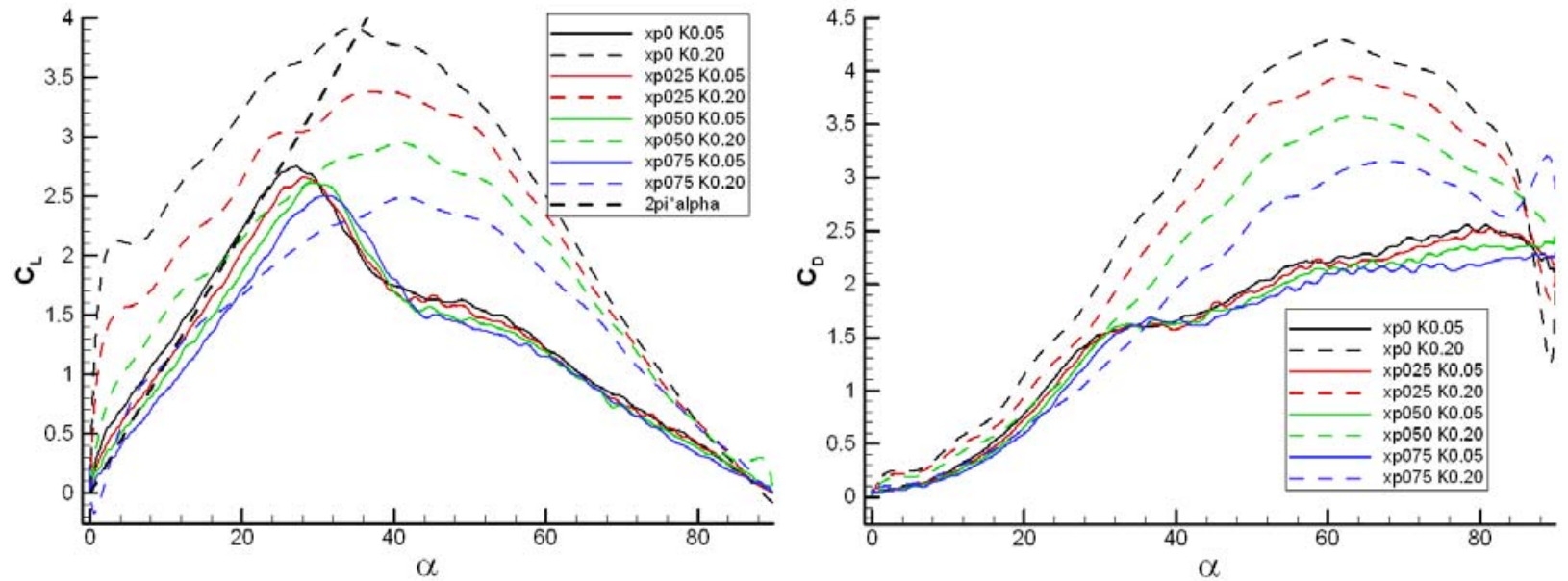

Figure 8. Survey of pitch pivot point location $(x / c=0,0.25,0.50$ and 0.75$)$ for $K=0.05$ and $0.20 ; \operatorname{Re}=20 K$.
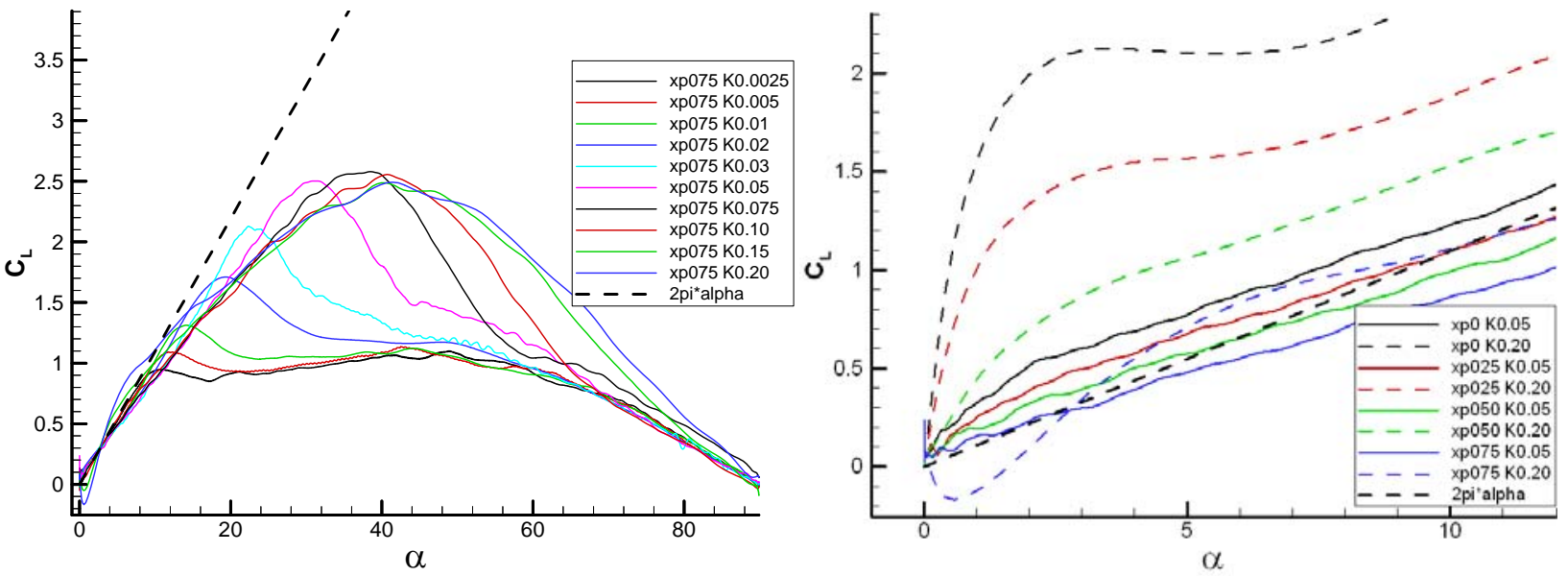

Figure 9. Trends in lift coefficient due to pivot point effects. Lift vs. reduced frequency for $\mathbf{x} / \mathbf{c}=0.75$ pivot (left), and comparisons of lift coefficient vs. pivot point location for $K=0.05$ and 0.20 at low angle of attack (right).

The third cause of trends in force coefficient vs. pivot point location is noncirculatory effects. Moving the pivot point aft also reduces, and eventually reverses sign, of the noncirculatory contribution. For $\mathrm{K}=0.20$, the 
noncirculatory lift contribution is evidently absent for pivot at $\mathrm{x} / \mathrm{c}=0.50$, while for $\mathrm{x} / \mathrm{c}=0.75$, it is negative, as seen in the right-hand portion of Figure 9.

In sum, the further forward the pitch pivot point, the higher the force coefficients - both lift and drag. As a mechanism for both high lift production and deceleration, it is preferable therefore to pivot the wing at as far forward a point as possible.

\section{Role of Streamwise Deceleration}

All of the aforementioned results were in conditions of steady free-stream, where the "perching" motion degenerated to a conventional pitch about a fixed pivot point. This is the natural experiment in a wind tunnel or water tunnel. Here we use a linear deceleration profile in the streamwise or "surge" direction, where the model decelerates at constant rate from the initial nominal free-stream relative forward speed at $\alpha=0^{\circ}$, to zero relative freestream at $\alpha=90^{\circ}$. Lift and drag curves for $\mathrm{K}=0.01,0.03,0.05,0.10$ and 0.20 with and without surge are shown for pivot points $\mathrm{x} / \mathrm{c}=0.0,0.25,0.50$ and 0.75 in Figure 10, Figure 11, Figure 12 and Figure 13, respectively. Force coefficients are calculated with respect to the instantaneous dynamic pressure, from the nominal relative free-stream speed. Thus as $\alpha \rightarrow 90^{\circ}$, the nominal free-stream speed goes to zero. If there is any mismatch between tunnel speed and rig surge speed, a very slight nonzero effective free-stream results at $\alpha=90^{\circ}$, and aerodynamic force coefficients become infinite through what amounts to division by zero. Further, any errors in force tares will be amplified, as the small instantaneous dynamic pressure reduces balance signal to noise ratio. Small variations in aerodynamic force coefficients will appear as large oscillations. Lift coefficient becomes unreliable for $\alpha>80^{\circ}$ and drag coefficient is probably unreliable for $\alpha>70^{\circ}$. Nevertheless, one can elucidate the major trends of force coefficients with and without surge.

The importance of surge depends to some extent on pivot point location, as evidently reduction in streamwise speed couples into a force contribution depending on angle of attack rate. For pivot at $\mathrm{x} / \mathrm{c}=0.50$ and 0.75 , surge makes a minor difference in lift coefficient, except of course at the highest angles of attack. The contribution to drag coefficient difference is somewhat larger, because drag increases with increasing angle of attack, and the free-stream attenuation with surge is of course greater as angle of attack increases. For pivot point further upstream, surge plays a greater role. But even here the difference between with-surge and no-surge respective cases is perhaps surprisingly small, especially for $\alpha<30^{\circ}$ or so.

Surge is more important for high reduced frequency than for low frequency. For $\mathrm{K}=0.01$, there is essentially no surge effect, except for drag at $\alpha>60^{\circ}$. For $\mathrm{K}=0.20$, surge causes a proportionately much higher dependency, which again mainly manifests itself for $\alpha>30^{\circ}$. For $\mathrm{x} / \mathrm{c}=0.0$ pivot, lift and drag with surge are higher than without surge. As the pivot point is taken further aft, this relationship reverses, so that for pivot at $\mathrm{x} / \mathrm{c}=075$, the drag with-surge is much lower than without surge, while the comparable trend for lift is less acute.
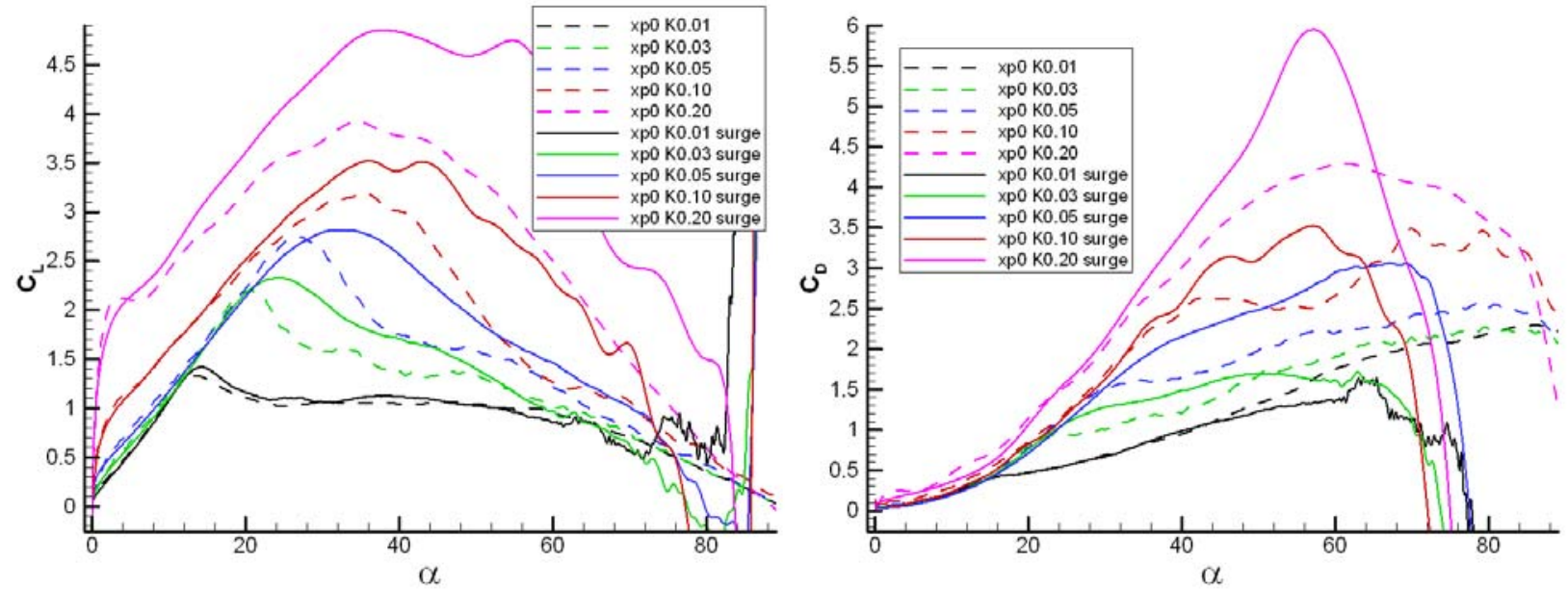

Figure 10. Comparison of surge (decelerating relative free-stream) vs. no-surge (steady relative free-stream) for a range of reduced frequencies, pivoting at $\mathbf{x} / \mathbf{c}=0.0$. Nominal Re based on initial free-stream is $20 \mathrm{~K}$. Lift (left) and drag (right). 

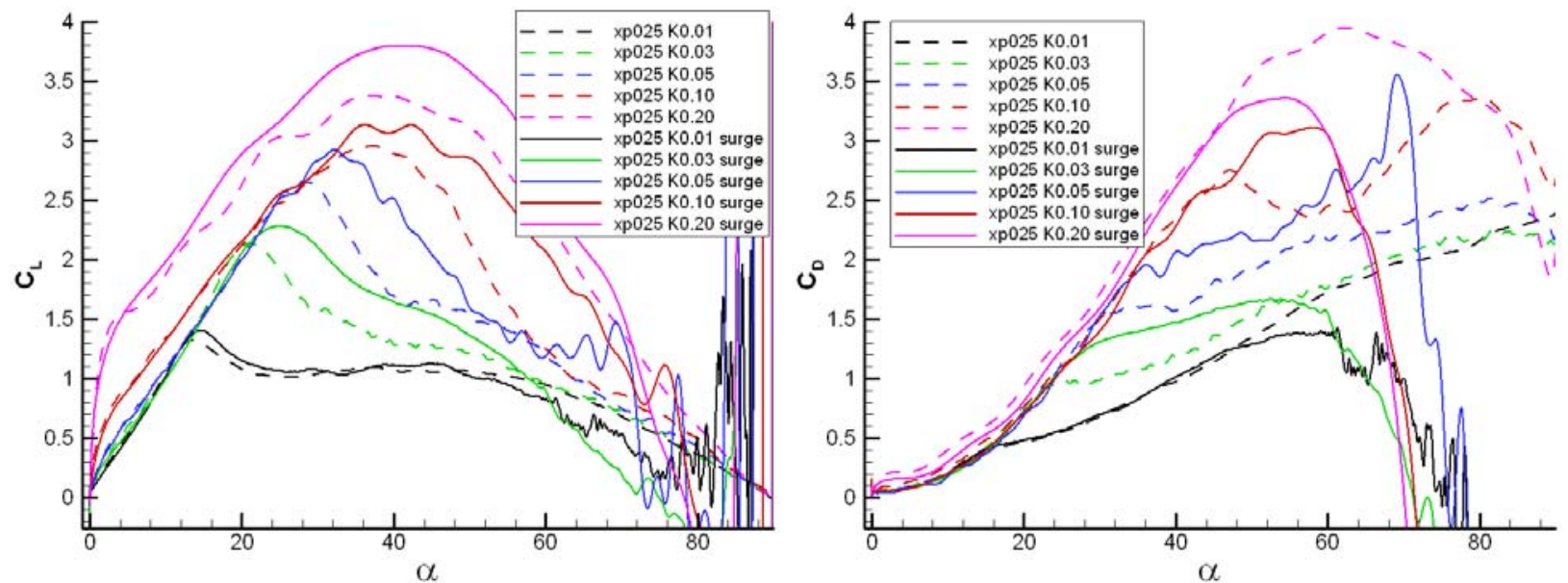

Figure 11. Comparison of surge (decelerating relative free-stream) vs. no-surge (steady relative free-stream) for a range of reduced frequencies, pivoting at $\mathbf{x} / \mathbf{c}=\mathbf{0 . 2 5}$. Nominal Re based on initial free-stream is $20 \mathrm{~K}$. Lift (left) and drag (right).
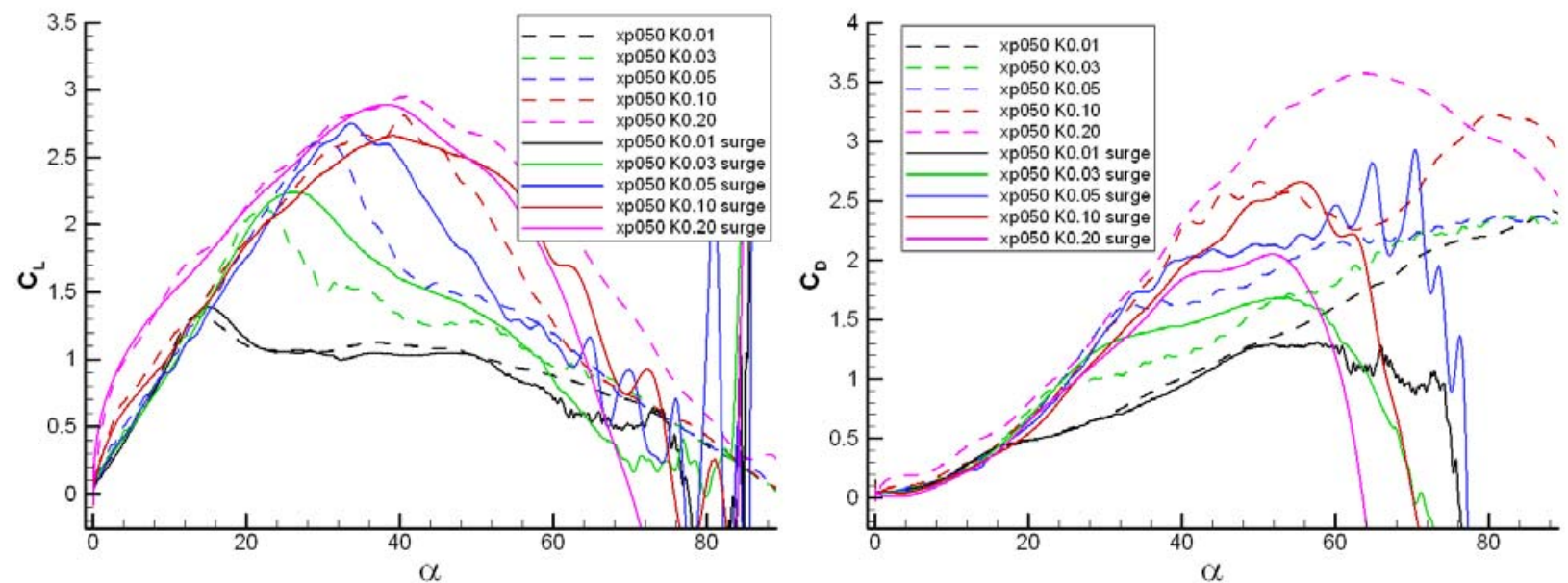

Figure 12. Comparison of surge (decelerating relative free-stream) vs. no-surge (steady relative free-stream) for a range of reduced frequencies, pivoting at $\mathbf{x} / \mathbf{c}=\mathbf{0 . 5 0}$. Nominal Re based on initial free-stream is $20 \mathrm{~K}$. Lift (left) and drag (right).
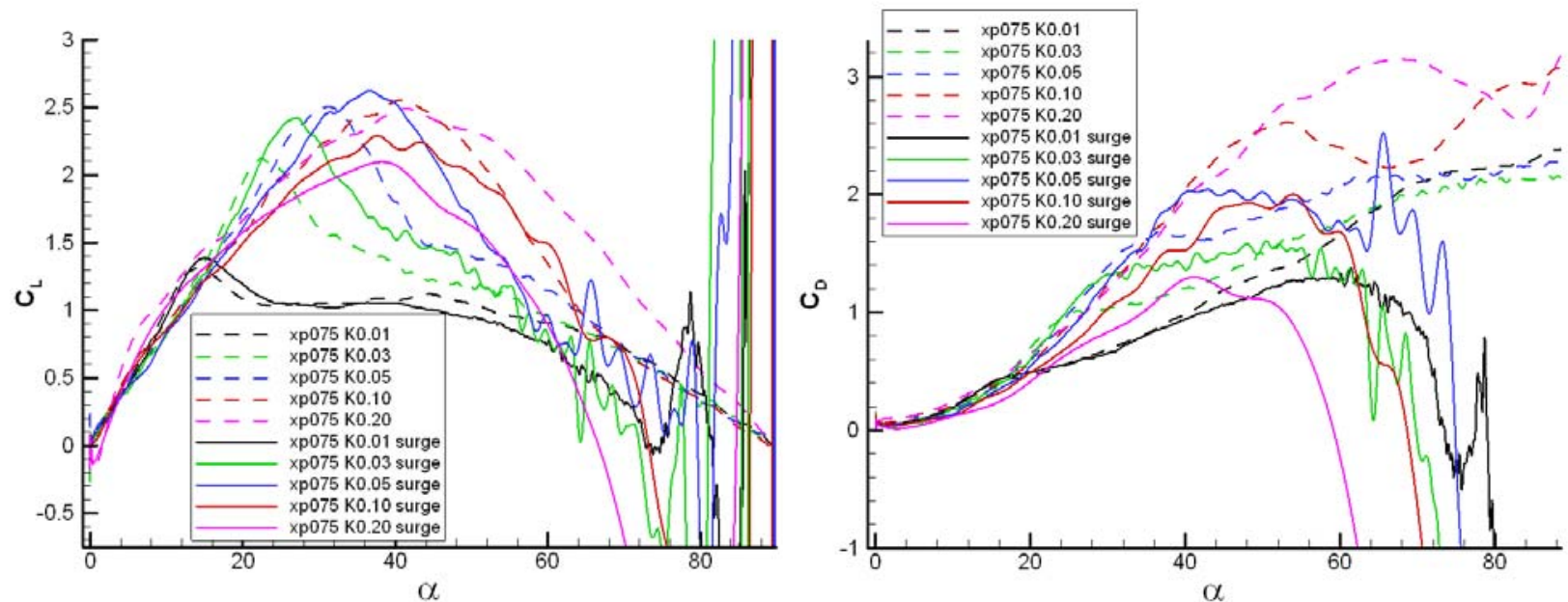

Figure 13. Comparison of surge (decelerating relative free-stream) vs. no-surge (steady relative free-stream) for a range of reduced frequencies, pivoting at $\mathbf{x} / \mathbf{c}=0.75$. Nominal Re based on initial free-stream is $20 \mathrm{~K}$. Lift (left) and drag (right). 
In sum, experiment in a maneuver that includes streamwise deceleration produces little departure from the case of steady free-stream for $\alpha<30^{\circ}$. But at higher angle of attack, there may be important trends, depending further on the pitch pivot point and on the reduced frequency.

\section{Connection between Flowfield Evolution and Aerodynamic Coefficient History for the Flat Plate}

As the flat plate is simpler than the SD7003 airfoil in terms of boundary layer physics and Reynolds number dependency, it is more straightforward to make observations of flowfield phenomena vs. the lift and drag. Comparison of the flat plate flowfield and the airfoil flowfield is a subject in its own right, and presumably the two will differ less and less as the reduced frequency of motion is increased. But presently we are more interested in general trends between the appearance of flowfield structures on the suction-side of the flat plate, and salient features in the lift and drag curves.

Figure 14 presents dye injection snapshots for $\mathrm{K}=0.20$ pivoting at $\mathrm{x} / \mathrm{c}=0.0$ and 0.75 , and $\mathrm{K}=0.03$ pivoting at $\mathrm{x} / \mathrm{c}=0.75$. Figure 15 compares $\mathrm{K}=0.01$ and $\mathrm{K}=0.03$ pivoting at $\mathrm{x} / \mathrm{c}=0.25$, and again $\mathrm{K}=0.03$ pivoting at $\mathrm{x} / \mathrm{c}=0.75$. All are for the 3 "-chord plate, at $\mathrm{Re}=20 \mathrm{~K}$, with constant free stream. $\mathrm{K}=0.01$ shows no evidence of LEV formation. Peak lift is attained at $\alpha \sim 13^{\circ}$, shortly after the first row in Figure 15. The lack of LEV formation is consistent with adherence to the classical 2D lift curve, $C_{L}=2 \pi \alpha$. As expected from the airfoil data in Figure 3 , at $\mathrm{K}=0.03$ there is formation of a structure akin to an LEV, but it is more diffuse for the plate than for the airfoil. There is also a clear role of pivot point effects: the further aft the pivot point, the more delayed the LEV formation and shedding. In the middle row of Figure 15 , for $\mathrm{K}=0.03$ with pivot at $\mathrm{x} / \mathrm{c}=0.25$, the LEV has already been ejected by $\alpha=22.5^{\circ}$, at the second row of images. For $\mathrm{K}=0.03$ with $\mathrm{x} / \mathrm{c}=0.75$ pivot point, however, the LEV is near its peak size. This delay in LEV formation, attainment of maximal size and eventual ejection, is consistent with the lift coefficient trends in Figure 8: the further aft the pivot point, the higher the angle of attack at which peak lift occurs. This is also true from considering the $\mathrm{K}=0.20$ cases in Figure 14. However, it is not clear whether the $\mathrm{LEV}$ for $\mathrm{K}=0.20$ pivoting at $\mathrm{x} / \mathrm{c}=0.0$ is stronger than for the 0.75 -pivot case. For 0.0 pivot, the LEV is larger sooner, and possibly this makes a larger lift contribution because the LEV-associated suction projects more in the lift direction at lower plate geometric incidence angle. But the eventual size of the LEV in the two $\mathrm{K}=0.20$ case is comparable. The main difference is in the TEV, which is much larger for the 0.0-pivot case. There is also a difference in the passage from motion-induce vortex shedding to the eventual static bluff-body Karman shedding at ensues after the plate reaches $\alpha=90^{\circ}$

The role of LEVs in lift coefficient history vs. angle of attack is a subject attracting much attention. "Vortical lift" can be important, in the sense of extra lift from organized leading-edge separation, beyond that attainable from attached flow, in the lifting-line or lifting-surface sense. Polhamus' suction analogy and its generalization by Lamar $^{19}$ are the generally-cited operative concepts. Unfortunately neither a force balance measurement nor a dye injection sequence yield sufficient information to decompose lift into vortical and nonvortical parts. To verify such a decomposition from experimental data, one could for example calculate the lift from flowfield velocity and vorticity data ${ }^{20}$, then subtract the LEV's vorticity contribution from the flowfield, and calculate an alternative lift to isolate the LEV's contribution. But an alternative viewpoint is that it is not necessarily the case that the LEV generates a large lift increment by itself, but rather, it is responsible for regularizing the flow separation such that lift in the sense of $\mathrm{C}_{\mathrm{L}}=2 \pi \alpha$ is possible even at high angle of attack. The evidence is that for moderate reduced frequencies, where rate-effects are unimportant, marked departure from $\mathrm{C}_{\mathrm{L}}=2 \pi \alpha$ occurs precisely in the region of angle of attack where the LEV is ejected. Further, there is no additional increment in lift centered about the angle of attack region where the LEV forms and attains its maximal size.

The dye injection images also resolve what might be termed the starting vortex, visible for example for, $22.5^{\circ}$ for the $\mathrm{K}=0.20$ LE-pivot case in Figure 14. Circulation from the starting vortex, in opposite sense to that of the bound vorticity or the LEV, should reduce net lift. But this is not discernable from the corresponding lift plot (Figure 8); that is, there is not a relaxation-time over which there is a lift decrement due to the starting vortex.

Lastly, we return to the 6"-chord plate to compare flowfield evolution between it and the 3"-chord plate, for pitch of $0^{\circ}$ to $90^{\circ}, \mathrm{K}=0.03$, pivot about $\mathrm{x} / \mathrm{c}=0.25$ (Figure 16). The comparison is somewhat crude because of different camera viewing angles of the two cases, and because the 6" plate did not have TE dye injection. Nevertheless, not great difference in flowfield evolution is apparent. The leading-edge flow separation region for the 6" plate at $\alpha=16.8^{\circ}$ engulfs less of the plate suction side than for the corresponding 3"-chord case, and at $\alpha=22.5^{\circ}$ the 6"-plate still shows an LEV-like separation on the suction side, while the 3" plate is entering a deep-stall type of flow separation. But at $\alpha=45^{\circ}$, where the two plates diverge maximally in measured lift coefficient, the two respective flowfields are much alike. It thus appears that blockage manifests itself more in the force data than in the flowfield evolution. 


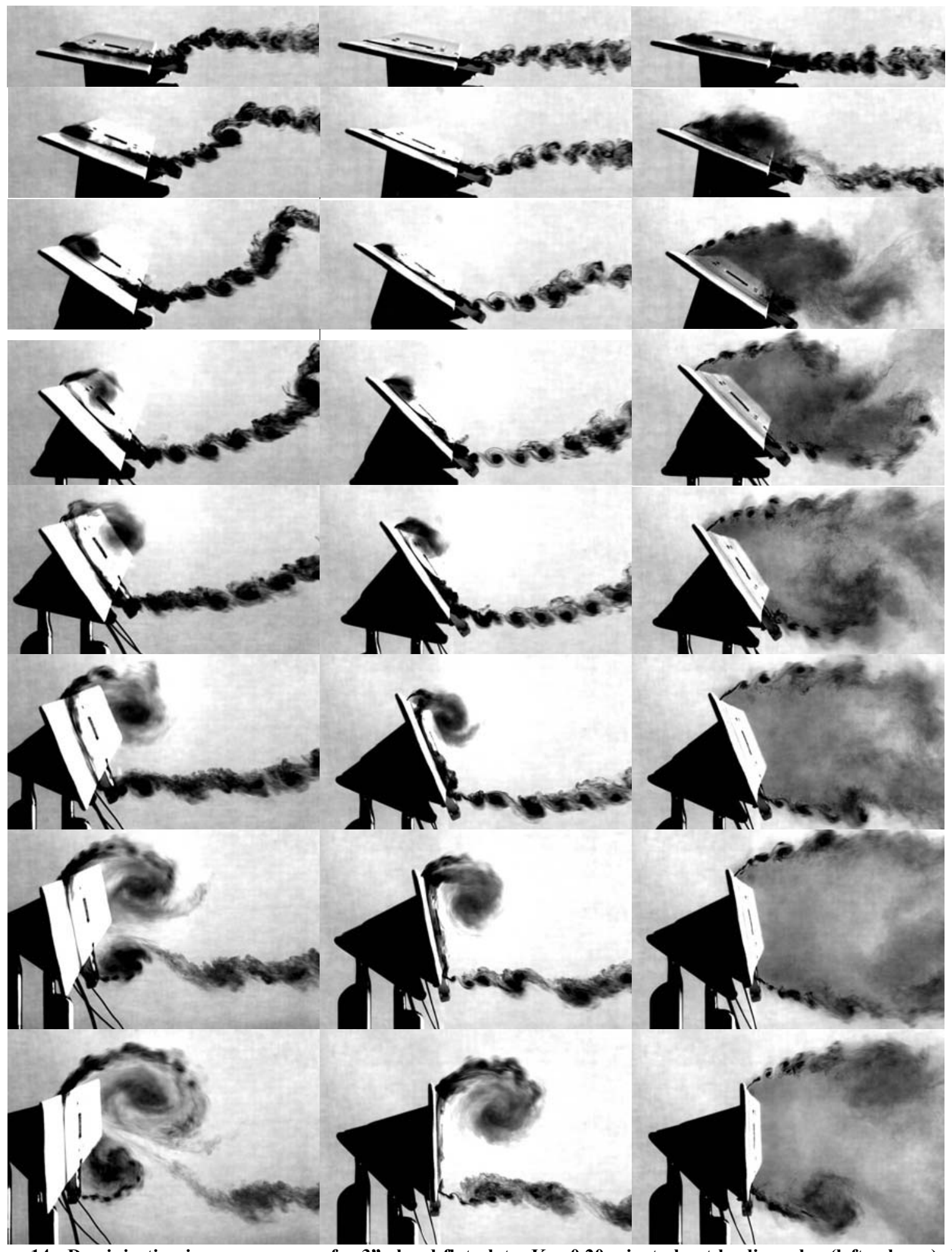

Figure 14. Dye injection image sequences for 3 "-chord flat plate: $K=0.20$, pivot about leading edge (left column); $K=$ 0.20 , pivot about $\mathrm{x} / \mathrm{c}=0.75$ (middle column); and $\mathrm{K}=0.03$, pivot about $\mathrm{x} / \mathrm{c}=\mathbf{0 . 7 5}$ (right column). Snapshots taken at $\alpha=$ $11.2^{\circ}, 22.5^{\circ}, 33.7^{\circ}, 45^{\circ}, 56.3^{\circ}, 67.6^{\circ}, 78.9^{\circ}$ and $90^{\circ}$. 


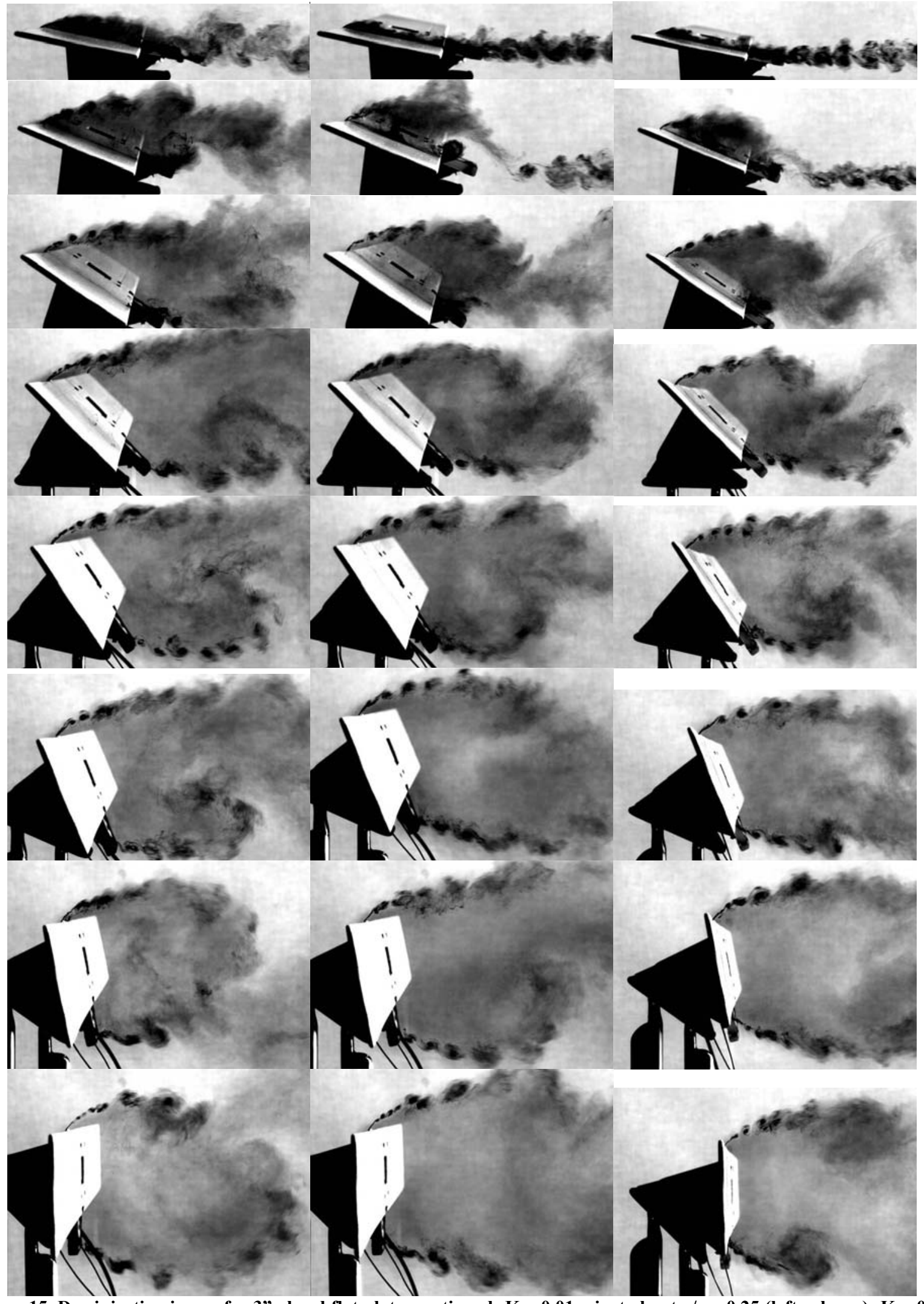

Figure 15. Dye injection image for 3"-chord flat plate, continued: $K=0.01$, pivot about $x / c=0.25$ (left column); $K=0.03$, pivot about $\mathrm{x} / \mathrm{c}=0.25$ (middle column); and repeat from Figure 14 of $\mathrm{K}=\mathbf{0 . 0 3}$, pivot about $\mathrm{x} / \mathrm{c}=0.75$ (right column).

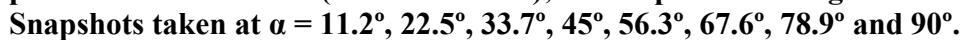




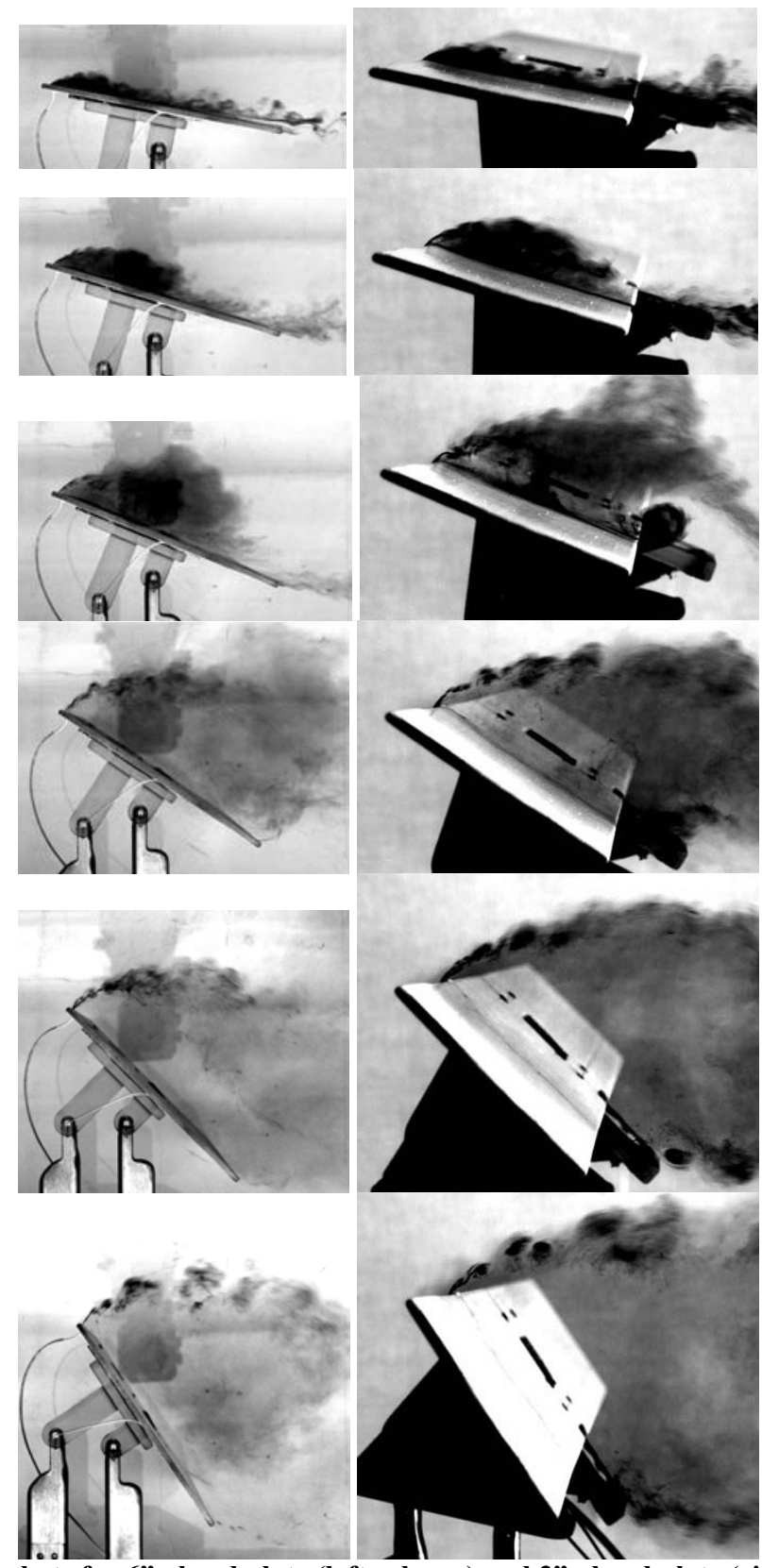

Figure 16. Dye injection snapshots for 6"-chord plate (left column) and 3"-chord plate (right column), $\mathrm{K}=\mathbf{0 . 0 3}$, pivot at $\mathrm{x} / \mathrm{c}=0.25$. Snapshots taken at $\alpha=11.2^{\circ}, 16.8^{\circ}, 22.5^{\circ}, 33.7^{\circ}, 45^{\circ}, 56.3^{\circ}$, and $67.6^{\circ}$.

\section{Conclusion}

We abstract the maneuver of "perching" as a linear pitch ramp of nominally $2 \mathrm{D}$ plates and airfoils from either $0^{\circ}$ to $45^{\circ}$ or $0^{\circ}$ to $90^{\circ}$, both with and without a streamwise deceleration from the initial free-stream speed to zero. Reduced frequencies of interest ranged from $\mathrm{K}=0.0025$ through 0.20 , and pivot points from $\mathrm{x} / \mathrm{c}=0.0$ through 0.75 were considered. The resulting parameter study implies essentially three regimes of unsteadiness, depending on the reduced frequency. For $\mathrm{K}<0.03$, the lift curve slope remains at the classical static value, but the stall angle is delayed with increasing reduced frequency. From $\mathrm{K} \sim 0.03$ and above, there is a nonlinear increase in lift curve slope at low angle of attack, and a smoothing of the stall peak, evidently due to a combination of rate-effects (first derivative of angle of attack) and the process of leading edge vortex formation, growth, saturation and ejection. At rates of $\mathrm{K} \sim 0.15$ and above, acceleration or noncirculatory effects are observed in those portions of the motion 
where acceleration is nonzero - that is, in the starting and stopping transients. Both rate-dependency and acceleration-dependency vary depending on pivot point location, and in general, both lift and drag are higher when the pivot point is further upstream. But the manifestation of unsteadiness is pervasive: even for $\mathrm{K}=0.005$, one observes an increase in peak lift coefficient beyond the static value.

From the computational viewpoint, to obtain good accuracy in general trends of lift and drag, even a 2D computation should suffice. To obtain the correct lift and drag variations in deep stall, some spanwise extent is imperative, as otherwise the leading edge separation will be incorrectly predicted. For full comparison with experiment, large spanwise extent - on the order of one chord - is desirable. As the computational spanwise extent was taken from 2D to 0.2 chords to 0.8 chords, the evinced leading and trailing vortical structures more closely resembled those of the particle image velocimetry experiment. Experiment, however, can be plagued with noise if there is no ensemble averaging, as in the present case. But dye injection results were an excellent qualitative proxy for vorticity derived from particle image velocimetry.

The role of surge, or decelerating effective free-stream, is intuitively important, since the effective reduced frequency of pitch becomes large at high angles of attack, when the free stream speed is low. However, the effect of surge on the lift and drag coefficients is quite small for $\alpha<30^{\circ}$ and only moderate for $30^{\circ}<\alpha<60^{\circ}$. At very high angles of attack there is a strong surge effect on aerodynamic coefficients, but only for high reduced frequency.

Finally, we note the role of blockage. For a ratio of model chord to test section height of 1:4, noticeable discrepancy in high angle of attack drag was observed. Halving this ratio by using a smaller-chord model returns the $\alpha=90^{\circ}$ drag to the accepted value, and this is our proxy for surmising that a 1:8-ratio experiment is acceptably blockage-free. Blockage is more significant in force data than in flowfield evolution, and much more significant in static force data than in the pitch-ramp cases.

\section{References}

${ }^{1}$ Carruthers, A.C. Thomas, A.L.R., and Taylor, G.K. "Automatic Aeroelastic Devices in the Wings of a Steppe Eagle Aquila Nipalensis", Journal of Experimental Biology, Vol. 210, 2007.

${ }^{2}$ Reich, G., and Wojnar, O., and Albertani, R. "Aerodynamic Performance of a Notional Perching MAV Design". AIAA 2009-0063.

${ }^{3}$ Graham, G.M., and Strickland, J.H. "An Experimental Investigation of an Airfoil Pitching at Moderate to High Rates to Large Angles of Attack". AIAA 1986-0008.

${ }^{4}$ Ol, M., Bernal, L., Kang, C.-K., and Shyy, W. "Shallow and Deep Dynamic Stall for Flapping Low Reynolds Number Airfoils". Experiments in Fluids, Vol. 46, Issue 5, pp. 883-901, May 2009.

${ }^{5}$ Ol, M., McAuliffe, B. R., Hanff, E. S., Scholz, U., Kaehler, Ch., "Comparison of Laminar Separation Bubble Measurements on a Low Reynolds Number Airfoil in Three Facilities", AIAA 2005-5149.

${ }^{6}$ OL, M.V. "Vortical Structures in High Frequency Pitch and Plunge at Low Reynolds Number". AIAA-20074233.

${ }^{7}$ Willert, C.E., and Gharib, M. "Digital Particle Image Velocimetry," Experiments in Fluids, Vol. 10, No. 4, January, 1991.

${ }^{8}$ Visbal, M. and Gaitonde, D. "High-Order Accurate Methods for Complex Unsteady Subsonic Flows". AIAA Journal, Vol. 37, pp. 1231-1239, 1999.

${ }^{9}$ Visbal, M. and Gaitonde, D. "On the Use of High-Order Finite-Difference Schemes on Curvilinear and Deforming Meshes.”. Journal of Computational Physics, Vol. 181, pp. 155-185, 2002.

${ }^{10}$ Lele, S. K. "Compact Finite Difference Schemes with Spectral-like Resolution." Journal of Computational Physics, pp. 16-42, 1992.

${ }^{11}$ Albert, P. "Implicit Filtering in Conjunction with Explicit Filtering. Journal of Computational Physics, Vol. 44, pp. 212-219, 1981.

${ }^{12}$ Gaitonde, D. and Visbal, M. "Pade-Type Higher-Order Boundary Filters for the Navier-Stokes Equations." AIAA Journal, Vol. 38, pp. 2103-2112, 2000.

${ }^{13}$ Beam, R. and Warming, R. "An Implicit Factored Scheme for the Compressible Navier-Stokes Equations." AIAA Journal, Vol. 16, pp. 393-402, 1978.

${ }^{14}$ Pulliam, T. and Chaussee, D. "A Diagonal Form of an Implicit Approximate-Factorization Algorithm." Journal of Computational Physics, Vol. 17, 1981.

${ }_{15}$ Visbal, M. and Gaitonde, D. "Very High-Order Spatially Implicit Schemes for Computational Acoustics on Curvilinear Meshes." Journal of Computational Acoustics, Vol. 9, 2001. 
${ }^{16}$ OL, M., Altman, A., Eldredge, J., Garmann, D., and Lian, Y. "Summary of Progress on Pitching Plates: Canonical Problems in Low-Re Unsteady Aerodynamics". AIAA-2010-1085

${ }^{17}$ Hoerner, S.F. Fluid-Dynamic Drag. Hoerner Fluid Dynamics, PO Box 342, Brick Town, NJ, 08723, 1965.

${ }^{18}$ Leishman, J. G., Principles of Helicopter Aerodynamics. Cambridge Aerospace Series, 2002.

${ }^{19}$ Lamar, J. E.: "Extension of Leading-Edge-Suction Analogy to Wings with Separated Flow Around the Side Edges at Subsonic Speeds", NASA TR R-428, 1974.

${ }^{20}$ Baik, Y.S., Rausch, J., Bernal, L., Shyy, W., and Ol, M. "Experimental Study of Governing Parameters in Pitching and Plunging Airfoil at Low Reynolds Number". AIAA-2010-0388 